\title{
The NASA Astrophysics Data System: The search engine and its user interface
}

\author{
Guenther Eichhorn, Michael J. Kurtz, Alberto Accomazzi, Carolyn S. Grant, and Stephen S. Murray \\ Harvard-Smithsonian Center for Astrophysics, Cambridge, MA 02138, U.S.A. \\ Received August 30; accepted September 3, 1999
}

\begin{abstract}
The ADS Abstract and Article Services provide access to the astronomical literature through the World Wide Web (WWW). The forms based user interface provides access to sophisticated searching capabilities that allow our users to find references in the fields of Astronomy, Physics/Geophysics, and astronomical Instrumentation and Engineering. The returned information includes links to other on-line information sources, creating an extensive astronomical digital library. Other interfaces to the ADS databases provide direct access to the ADS data to allow developers of other data systems to integrate our data into their system.

The search engine is a custom-built software system that is specifically tailored to search astronomical references. It includes an extensive synonym list that contains discipline specific knowledge about search term equivalences.

Search request logs show the usage pattern of the various search system capabilities. Access logs show the worldwide distribution of ADS users.

The ADS can be accessed at:

http://adswww.harvard.edu
\end{abstract}

Key words: methods: data analysis - databases: misc publications, bibliography

\section{Introduction}

The Astrophysics Data System (ADS) provides access to the astronomical literature through the World Wide Web (WWW). It is widely used in the astronomical community. It is accessible to anybody world-wide through a forms based WWW interface. A detailed description of the history of the ADS is presented in the ADS Overview article (Kurtz et al. 2000, hereafter

Send offprint requests to: G. Eichhorn,

e-mail: gei@cfa.harvard.edu
OVERVIEW). The system contains information from many sources (journals, other data centers, individuals). A detailed description of the data that we get and how they are included in the ADS is presented in the ADS Data article (Grant et al. 2000, hereafter DATA). The incoming data are processed and indexed with custom-built software to take advantage of specialized knowledge of the data and the astronomical context. A description of this processing is given in the ADS Architecture article (Accomazzi et al. 2000, hereafter ARCHITECTURE). This article describes the development and the current status of the ADS Abstract Service user interface and search engine.

The ADS was created as a system to provide access to astronomical data (Murray et al. 1992). In 1993 the ADS started to provide access to a set of abstracts obtained from the NASA/STI (National Aeronautics and Space Administration/Scientific and Technical Information) project (Kurtz et al. 1993). The user interface was built with the proprietary software system that the ADS used at that time. The search engine of this first implementation used a commercial database system. A description of the system at that time is in Eichhorn 1994.

In 1994, the World Wide Web (WWW, www.w3.org 1999) became widely useful through the NCSA Mosaic Web Browser (Schatz \& Hardin 1994). The design of the ADS Abstract Service with a clean separation between the user interface and the search engine made it very easy to move the user interface from the proprietary ADS system to the WWW. In February 1994, a WWW interface to the ADS Abstract Service was made available publicly. The WWW interface to the ADS is described by Eichhorn et al. (1995b) and Eichhorn et al. (1995a). Within one month of the introduction of the WWW interface, the usage of the Abstract Service tripled, and it has continued to rise ever since (Eichhorn 1997).

With the increased usage of the system due to the easy access through the WWW, severe limitations of the underlying commercial database system very quickly 
became apparent. We soon moved to an implementation of the search engine that was custom-built and tailored to the specific requirements of the data that we used.

In January 1995 we started to provide access to scanned journal articles (Accomazzi et al. 1996). The user interface to these scans provided the user with the capability to access the scans in various formats, both for viewing and for printing.

With time, other interfaces to the abstracts and scanned articles were developed to provide other data systems the means to integrate ADS data into their system (Eichhorn et al. 1996b).

With the adoption of the WWW user interface and the development of the custom-built search engine, the current version of the ADS Abstract Service was basically in place. The following sections describe the current status of the different access capabilities (Sects. 2 and 3), the search engine (Sects. 4 and 5), access statistics for the ADS system (Sect. 6), and future plans for the ADS interface and search engine (Sect. 7).

\section{Data access}

The ADS services can be accessed through various interfaces. Some of these interfaces use WWW based forms, others allow direct access to the database and search system through Application Program Interfaces (APIs). This section describes the various interfaces and their use, as well as the returned results.

\subsection{Forms based interfaces}

\subsubsection{Abstract service}

a. User interface

The main query forms (Figs. 1, 2, 3) provide access to the different abstract databases. These forms are generated on demand by the ADS software. This allows the software to check the user identification through the HTTP (HyperText Transfer Protocol) cookie mechanism (see Sect. 3), so that the software can return a customized query form if one has been defined by the user. It also adapts parts of the form according to the capabilities of the user's web browser.

The query form allows the user to specify search terms in different fields. The input parameters in each query field can be combined in different ways, as can the results obtained from the different fields (Fig. 1). The user can specify how the results are combined through settings on the query form (Fig. 3). The combined results can then be filtered according to various criteria (Fig. 2).

The database can be queried for author names, astronomical objects names, title words, and words in the abstract text. References can be selected according to the publication date. The author name, title, and text

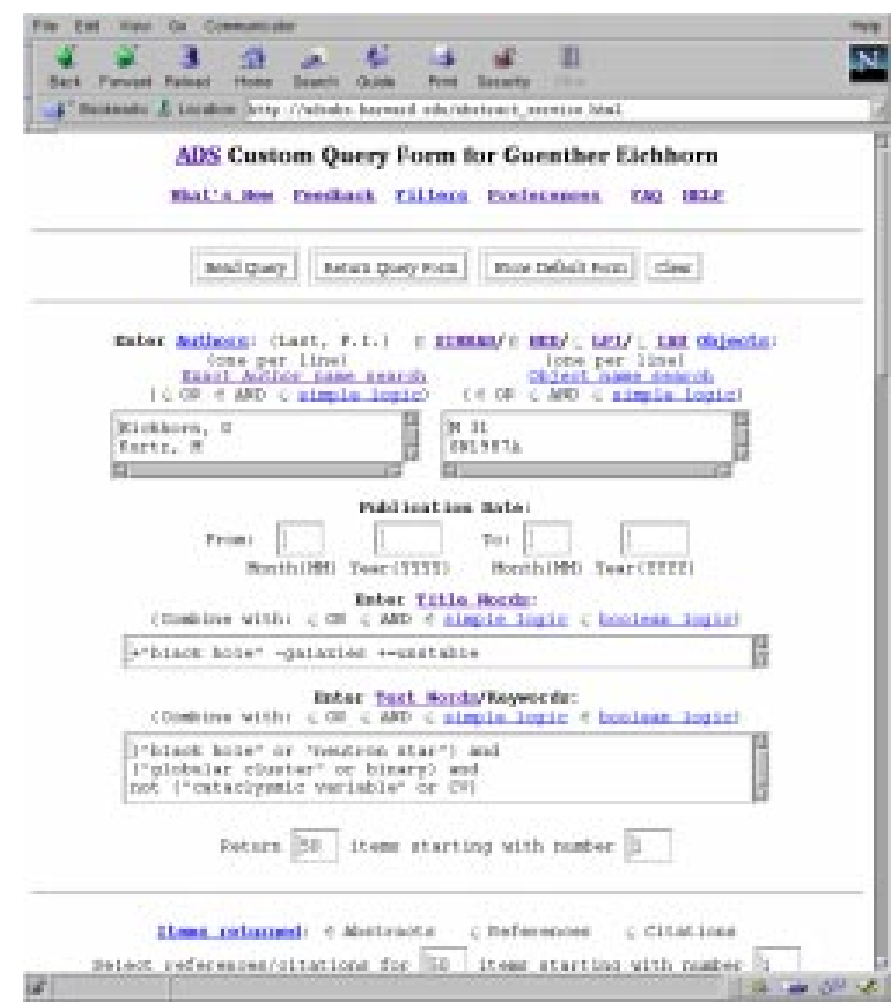

Fig. 1. The ADS Abstract Service query form provides the capability to query the database by authors, object names, title and text words

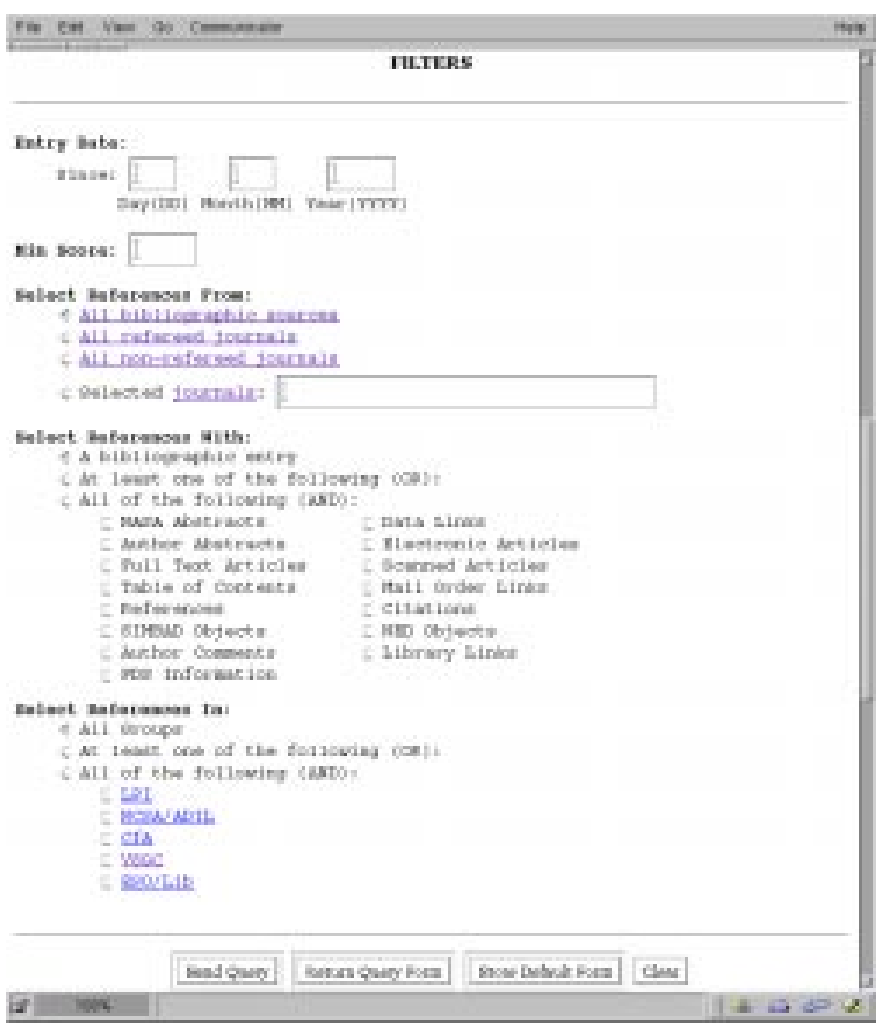

Fig. 2. The Filter section of the query form allows selection of references that have specific properties 


\begin{tabular}{|c|c|c|c|c|c|}
\hline \multicolumn{6}{|c|}{ 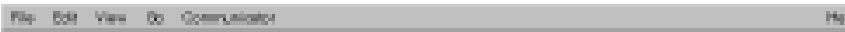 } \\
\hline & Brod Doner & Petare Dover & atore & 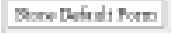 & chen \\
\hline \multicolumn{6}{|c|}{ SORTINE } \\
\hline \multicolumn{6}{|c|}{ 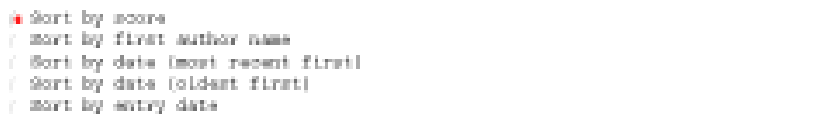 } \\
\hline \multicolumn{6}{|c|}{ SETTINGS } \\
\hline & nether: & stjectr & Fitle & watract & \\
\hline 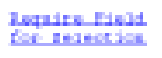 & & & ᄃ & & \\
\hline 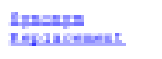 & s & & * & $s$ & \\
\hline Ielatins llatita & $3=1$ & 3.1 & $9=3$ & 9.1 & \\
\hline $\begin{array}{l}\text { me res } \\
\text { Mulghting }\end{array}$ & s & s & v & s & \\
\hline $\begin{array}{l}\text { mianed } \\
\text { ioscing }\end{array}$ & $c$ & & s & s & \\
\hline a $\quad k$ & & & & & 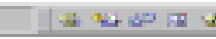 \\
\hline
\end{tabular}

Fig. 3. The Settings section of the query form allows the user to customize the search

fields are case insensitive. The object field is case sensitive when the IAU (International Astronomical Union) Circulars (IAUC) object name database is searched, since the IAU object names are case sensitive. In the author and object name fields, the form expects one search term per line since the terms can contain blanks. In the title and text fields line breaks are not significant.

Author Name Field The author names are indexed by last name and by a combination of last name and first initial, separated by a comma. To account for differences in the spelling of the same author name, the search system contains a list of author names that are spelled differently but are in fact names of the same author. This allows for automatically retrieving all versions of common spelling differences. This is useful for instance for German umlaut spelled as Muller and Mueller, or variations in the transliteration of names from non-English alphabets like Cyrillic. An example of such an entry in the author synonym list is:
AFANASJEV, $\mathrm{V}$
AFANAS'EV, $V$
AFANAS' IEV, $V$
AFANASEV, $V$
AFANASYEV, $V$
AFANS'IEV, $V$
AFANSEV, $V$

Without this synonym replacement capability, author searches would obviously be much less effective. On user request we also include name changes (e.g. due to marriage) in the author synonym list. Combinations of search results within the author field use "OR", "AND", or simple logic (see below), depending on user selection.

Author names are quite often spelled differently in different publications. First names are sometimes spelled out, sometimes only first initials are given, and sometimes middle initials are left out. This makes it impossible to index all different spellings of a name together automatically.

To handle these different requirements, author names are indexed three times, once with the last name only, once with the last name and first initial, and once with the complete name as it is specified in the article.

To access these different indexes, we provide two user interfaces for author queries. The regular user interface allows the user to search for either a last name or a last name combined with the first initial. This allows for fairly discriminating author searches. It is a compromise between the need to discriminate between different authors, and the need to find all instances of a given author. It identifies all different versions of a given author quite reliably, but it indexes together different authors with the same first initial. For cases where this search method is not discriminating enough, we provide a second user interface to the index of the full names, which does not attempt to index different spellings of the same author together. When the user selects "Exact Author Search" and specifies an author's last name or last name and first initial, a form is returned with all distinct full author names that match the specified name. The user then selects all the different spellings of the desired name and queries the database for articles that contain any one of these different versions of an author's name. For instance specifying:

Eichhorn, G

in the exact author name form returns the list:

\section{EICHHORN, G.}

EICHHORN, GERHARD

EICHHORN, GUENTHER

EICHHORN, GUNTHER

Selecting the first, third, and fourth author name from that list will return all articles by the first author of this article. Any articles by the second author containing only the first initial will also be returned, but this is unavoidable.

Object Name Field This field allows the user to query different databases for references with different astronomical objects. The databases that provide object information are: SIMBAD (Set of Identifications, Measurements and Bibliographies for Astronomical Data) at the Centre des Données Astronomique de Strasbourg (CDS), France 
(Egret et al. 1991); the NASA Extragalactic Database (NED) at the Infrared Processing and Analysis Center (IPAC), Jet Propulsion Laboratory (JPL), Pasadena, CA (Madore et al. 1992); the IAU Circulars (IAUC) and the Minor Planet Electronic Circulars (MPEC), both provided by the Central Bureau for Astronomical Telegrams (CBAT) at the Harvard-Smithsonian Center for Astrophysics in Cambridge, MA (Marsden 1980); and a database with objects from publications from the Lunar and Planetary Institute (LPI) in Houston (mainly Lunar sample numbers and meteorite names). The user can select which of these databases should be queried. If more than one database is searched, the results of these queries are merged. The LPI database does not have any entries in common with the other databases. The SIMBAD, NED, and IAUC databases sometimes have information about the same objects.

Title and Abstract Text Fields These fields query for words in the titles of articles or books, and in the abstracts of articles or descriptions of books respectively. The words from the title of each reference are also indexed in the text field so they will be found through either a title or a text search. Before querying the database the input in these fields is processed as follows:

1. Apply translation rules. This step merges common expressions into a single word so that they are searched as one expression. Regular expression matching is used to convert the input into a standard format that is used to search the database. For instance $M 31$ (with a space) is translated to M31 (without a space) for searching as one search term. In order to make this general translation, a regular expression matching and substitution is performed that translates all instances of an "M" followed by one or more spaces or a hyphen followed by a number into "M" directly followed by the number. Other translation rules include the conversion of NGC 1234 to NGC1234, contractions of $T$ Tauri, Be Star, Shoemaker Levy, and several others (see ARCHITECTURE).

2. Remove punctuation. In this step all nonalphanumeric characters are removed, unless they are significant (for instance symbols used in the simple logic (see below), "+" and "-" before numbers, or "." within numbers).

3. Translate to uppercase. All information in the index files is in uppercase, except for object names from the IAU Circulars.

4. Remove kill words. This step removes all nonsignificant words. This includes words like "and", "although", "available", etc. (for more details see ARCHITECTURE).

In the title and text fields, searching for phrases can be specified by enclosing several words in either single or double quotes, or concatenating them with periods (".") or hyphens ("-"). All these accomplish the same goal of searching the database for references that contain specified sequences of words. The database is indexed for twoword phrases in addition to single words. Phrases with more than two words are treated as a search for sets of two-word phrases containing the first and second word in the first phrase, the second and third word in the second phrase, etc.

\section{b. Searching}

After the search terms are pre-processed, the databases of the different fields are searched for the resulting list of words, the results are combined according to the selected combination rules, and the resulting score is calculated according to the selected scoring criteria. These combination rules provide the means for improving the selectivity of a query.

Search Word Selection The database is searched for the specified words as well as for words that are synonymous with the specified term. One crucial part to successful searches in a free text search system is the ability to not only find words exactly as specified, but also similar words. This starts with simply finding singular and plural forms of a word, but then needs to be extended to different words with the same meaning in the normal usage of words in a particular field of science. In Astronomy for instance "spectrograph" and "spectroscope" have basically the same meaning and both need to be found when one of these words is specified in the query. Even further reaching, more discipline-specific synonyms are necessary for efficient searches such as "metallicity" and "abundance" which have the same meaning in astronomical word usage. In order to exhaustively search the database for a given term, it is important to search for all synonyms of a given word. The list of synonyms was developed manually by going through the list of words in the database and grouping them according to similar meanings. This synonym list is a very important part of the ADS search system and is constantly being improved (see ARCHITECTURE).

The list of synonyms also contains non-English words associated with their English translations. These words came from non-English reference titles that we included in the database. This allows searches with either the English or non-English words to find references with either the English word or the non-English translation. We are in the process of extending this capability by including translations of most of the words in our database into several languages (German, French, Italian, Spanish). This will allow our users to phrase queries in any of these languages. We expect to complete this project sometime in 2000.

By default a search will return references that contain the search word or any of its synonyms. The user can choose to disable this feature if for some reason a specific word needs to be found. The synonym replacement 
can be turned off completely for a field in the "Settings" section of the query form. This can be used to find a rare word that is a synonym of a much more frequent word, for instance if you want to look for references to "dateline", which is a synonym to "date". Synonym replacement can also be enabled or disabled for individual words by prefixing a word with "=" to force an exact match without synonym replacement. When synonym replacement is disabled for a field, it can be turned on for a particular word by prefixing it with "\#".

Selection Logic Within a Field There are four different types of combinations of results for searches within a field possible.

1. OR

2. AND

3. Simple logic

4. Full boolean logic

1. Combination by "OR": The resulting list contains all references that contain at least one of the search terms.

2. Combination by "AND": The resulting list has only references that contain every one of the search terms.

3. Combination by simple logic: The default combination in this logic is by "OR". Individual terms can be either required for selection by prefixing them with a "+", or can be selected against by prefixing them with a "-". In the latter case only references that do not contain the search term are returned. If any of the terms in the search is prefixed by a "+", any other word without a prefix does not influence the resulting list of references. However, the final score (see below) for each reference will depend on whether the other search terms are present.

4. Combination by full boolean logic: In this setting, the user specifies a boolean expression containing the search terms and the boolean operators "and", "or", and "not", as well as parentheses for grouping. A boolean expression could for instance look like:

(pulsar or "neutron star") and ("red shift" distance) and not 1987A.

This expression searches for references that contain either the word pulsar or the phrase "neutron star" and either the phrase "red shift" or the word distance ("or" being the default), but not the word 1987A.

Selection Logic Between Fields In the settings part of the query form, the user can specify fields that will be required for selection. If a field is selected as "Required for Selection" only references that were selected in the search specified in that field will be returned. If one field is selected as "Required for Selection", the searches in fields that are not set as "Required for Selection" do not influence the resulting list, but they influence the final score. c. Scoring

The list of references resulting from a query is sorted according to a "score" for each reference. This score is calculated according to how many of the search items were matched. The user has the choice between two scoring algorithms:

\section{1. proportional scoring}

2. weighted scoring

These scoring algorithms have been analyzed by Salton \& McGill (1983).

In proportional scoring, the score is directly proportional to the number of terms found in the reference. In weighted scoring, the score is proportional to the inverse logarithm of the frequency of the matched word. This weighting gives higher scores for words that are less frequent in the database and therefore presumably more important indicators of the relevance of a match. In the settings section of the query form the user can select which type of scoring should be used for each query field separately. The default setting for title and text searches is the weighted scoring. For author searches proportional scoring is the default. Once the score for each query field is calculated, the scores are normalized so that a reference that matches all words in a field receives a score of 1.

The normalized scores from the different fields are then combined to calculate a total score. Again the result is normalized so that a reference that matches all words in each query field has a score of 1 . The user can influence this combining of scores from the different search fields by assigning weights to the different fields. This allows the user to put more emphasis in the selection process on, for instance, the object field by assigning a higher weight to that field. Another use of the weight field is to select against a field. For instance specifying an object name and an author name and selecting a negative weight to the author field will select articles about that object that were not written by the specified author.

The relative weights for the different search fields can be set by the user. The ADS provides default weights as follows:
Authors: 1.0
Objects: 1.0
Title: 0.3
Text: $\quad 3.0$

These default weights were determined on theoretical grounds, combined with trial and error experimentation. We used different search inputs from known research fields and different weights and ranked the resulting lists according to how well they represented articles from these research fields. The weights listed above gave the best results.

\section{d. Filtering of selected references}

The selected references can be filtered according to different criteria (see Sect. 4.5) in order to reduce the number of 


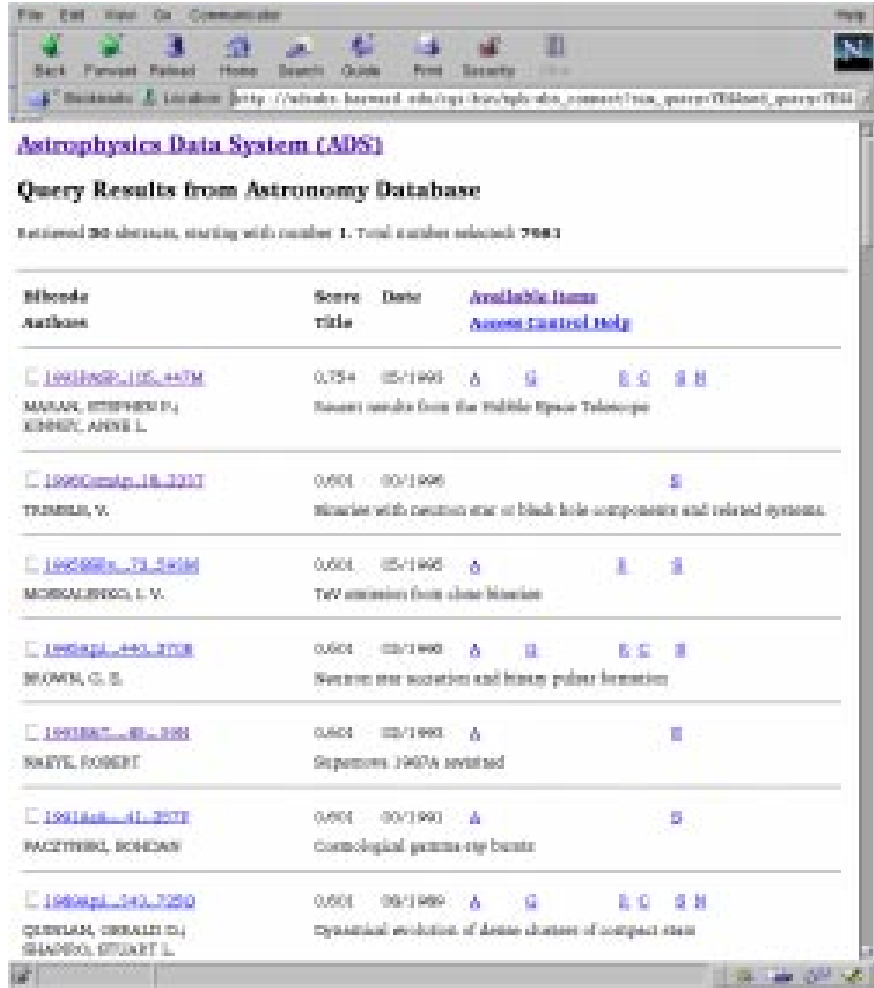

Fig. 4. Entries in the list of references returned by an ADS query contain the bibliographic code (1) the matching score (2), the publication date (3), a list of data links (4), the list of authors (5), and the title of the reference (6)

returned references. The user can select references according to their entry date in the database, a minimum score (see above), the journal they are published in, whether they have pointers to selected external data sources, or whether they belong to one or more of several groups of references. This allows a user for instance to select only references from refereed journals or from one particular journal by specifying its abbreviation. It also allows a user to select only references that have links to external data sets, on-line articles, or that have been scanned and are available through the ADS Article Service.

e. Display of search results

The ADS system returns different amounts of information about a reference, depending on what the user request was. This section describes the different reference formats.

Short Reference Display The list of references returned from a query is displayed in a tabular format. The returned references are sorted by score first. For equal scores, the references are sorted by publication date with the latest publications displayed first.

A typical reference display is shown in Fig. 4. The fields in such a reference are shown in Fig. 5. They are as follows:

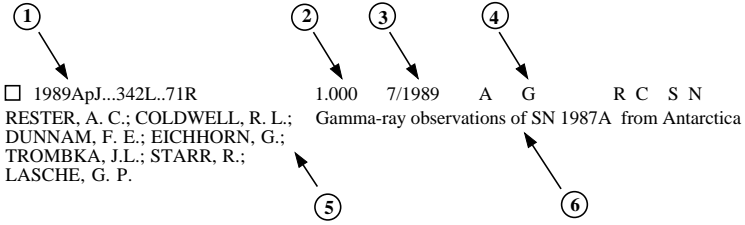

Fig. 5. Elements in the references returned from an ADS query

1. Bibliographic Code: This code identifies the reference uniquely (see DATA and Schmitz et al. 1995). Two important properties of these codes are that they can be generated from a regular journal reference, and that they are human readable and can be understood and interpreted.

2. Score: The score is determined during the search according to how well each reference fits the query.

3. Date: The publication date of the reference is displayed as mm/yyyy.

4. Links: The links are an extremely important aspect of the ADS. They provide access to information correlated with the article. Table 1 shows the links that we currently provide when available.

A more detailed description of resources in the ADS that these links point to is provided in DATA.

Some of these links (for instance the "D" links) can point to more than one external information provider. In such cases the link points to a page that lists the available choices of data sources. The user can then select the more convenient site for that resource, depending on the connectivity between the user site and the data site.

5. Authors: This is the list of authors for the reference. Generally these lists are complete. For some of the older abstracts that we received from NASA/STI, the author lists were truncated at 5 or 10 authors, but every effort has been made to correct these abbreviated author lists (see DATA).

6. Title: The complete title of the reference.

The reference lists are returned as forms if table display is selected (see Sect. 3). The user can select some or all of the references from that list to be returned in any one of several formats:

i. HTML format: The HTML (HyperText Markup Language) format is for screen viewing of the formatted record.

ii. Portable Format: This is the format that the ADS uses internally and for exchanging references with other data centers. A description of this format is available online at:

\section{http://adsabs.harvard.edu/abs_doc/ abstract_format.html}

iii. BibTeX format: This is a standard format that is used to build reference lists for TeX (a typesetting language especially suited for mathematical formulas) formatted articles. 
Table 1. Links types and their numbers in the ADS database

\begin{tabular}{|c|c|c|}
\hline Link & Resource & Description \\
\hline A & Abstract & Full abstract of the article. These abstracts come from different sources. \\
\hline $\mathrm{C}$ & Citations & $\begin{array}{l}\text { A list of articles that cite the current article. This list is not necessarily complete (see } \\
\text { "R" References). }\end{array}$ \\
\hline $\mathrm{D}$ & On-line Data & Links to on-line data at other data centers. \\
\hline $\mathrm{E}$ & Electronic Article & $\begin{array}{l}\text { Links to the on-line version of the article. These on-line versions are in HTML format for } \\
\text { viewing on-screen, not for printing. }{ }^{\text {a }}\end{array}$ \\
\hline $\mathrm{F}$ & Printable Article & Links to on-line articles in PDF or Postscript format for printing. ${ }^{a}$ \\
\hline G & Gif Images & Links to the images of scanned articles in the ADS Article Service. \\
\hline I & Author Comments & $\begin{array}{l}\text { Links to author supplied additional information (e.g. corrections, additional references, } \\
\text { links to data). }\end{array}$ \\
\hline $\mathrm{L}$ & Library Entries & Links to entries in the Library of Congress on-line system. \\
\hline M & Mail Order & Links to on-line document delivery systems at the publisher/owner of the article. \\
\hline $\mathrm{N}$ & NED Objects & Access to lists of objects for the current article in the NED database. \\
\hline $\mathrm{O}$ & Associated Articles & $\begin{array}{l}\text { A list of articles that are associated with the current article. These can be errata or other } \\
\text { articles in a series. }\end{array}$ \\
\hline $\mathrm{P}$ & Planetary Data System & Links to datasets at the Planetary Data System. \\
\hline $\mathrm{R}$ & References & $\begin{array}{l}\text { A list of articles referred to in the current article. For older articles these lists are not nec- } \\
\text { essarily complete, they contain only references to articles that are in the ADS database. } \\
\text { For articles that are on-line in electronic form, the "R" link points to the on-line reference } \\
\text { list, and therefore the complete list of references in that article. }\end{array}$ \\
\hline $\mathrm{S}$ & SIMBAD Objects & Access to lists of objects for the current article in the SIMBAD database. \\
\hline $\mathrm{T}$ & Table of Contents & Links to the list of articles in a books or proceedings volume. \\
\hline $\mathrm{X}$ & Planetary Nebulae & Links to datasets in the Galactic Planetary Nebulae Database. \\
\hline
\end{tabular}

a There is generally access control at the site that serves these on-line articles.

iv. ASCII format: This is a straight ASCII text version of the abstract. All formatting is done with spaces, not with tabs.

v. User Specified Format: This allows the user to specify in which format to return the reference. The default format for this selection is the bibitem format from the AASTeX macro package. The user can specify an often used format string in the user preferences (see Sect. 3). This format string will then be used as the default in future queries.

The user can select whether to return the selected abstracts to the browser, a printer, a local file for storage, or email it to a specified address.

Full Abstract Display In addition to the information in the short reference list, the full abstract display (see Fig. 6) includes, where available, the journal information, author affiliations, language, objects, keywords, abstract category, comments, origin of the reference, a copyright notice, and the full abstract. It also includes all the links described above.

For abstracts that are displayed as a result of a search, the system will highlight all search terms that are present in the returned abstract. This makes it easy to locate the relevant parts in an abstract. Since the highlighting is somewhat resource intensive, it can be turned off in the user preference settings (see Sect. 3).

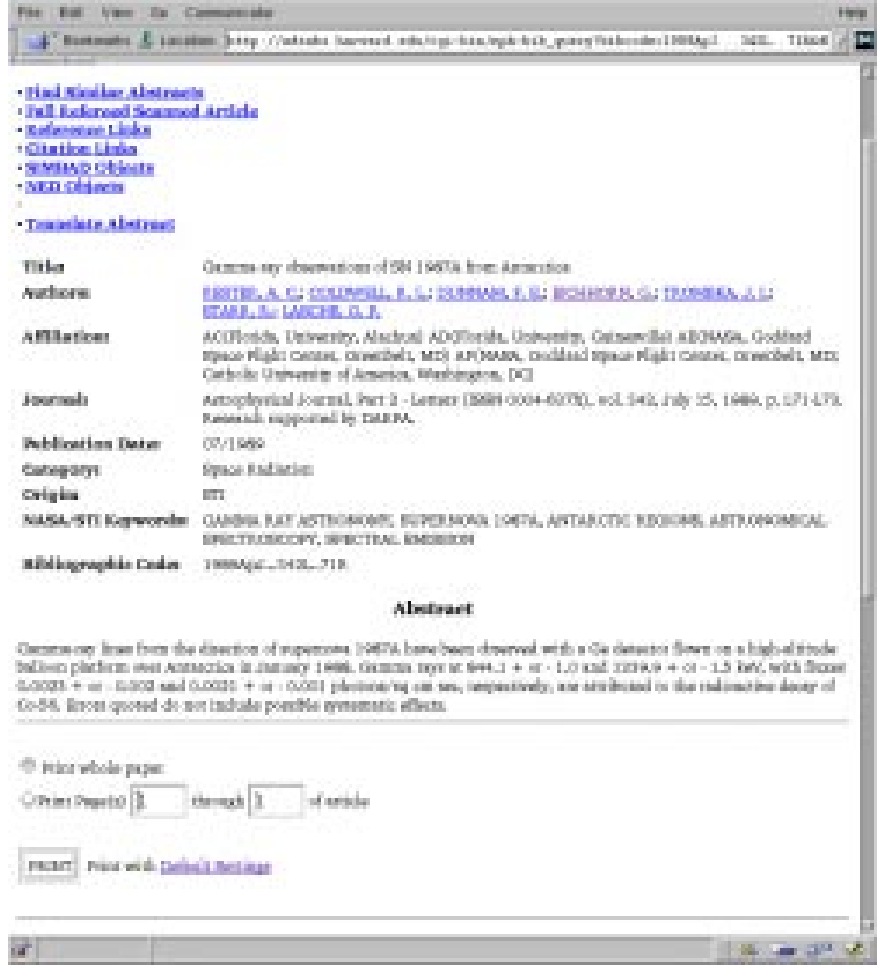

Fig. 6. The full abstract display contains (where available) the title, author list, journal information, author affiliations, publication date, keywords, the origin of the reference, the bibliographic code, the abstract, object names, abstract category, and a copyright notice 
For convenience, the returned abstract contains links that allow the user to directly retrieve the BibTex or the custom formatted version of the abstract.

The full abstract display also includes a form that provides the capability to use selected information from the reference to build a new query to find similar abstracts. The query feedback mechanism makes in-depth literature searches quick and easy. The user can select which parts of the reference to use for the feedback query (e.g. authors, title, or abstract). The feedback query can either be executed directly, or be returned as a query form for further modification before executing it, for instance to change the publication date range or limit the search to specific journals. This query feedback mechanism is a very powerful means to do exhaustive literature searches and distinguishes the ADS system from most other search systems. A query feedback ranks the database against the record used for the feedback and sorts it according to how relevant each reference is to the search record. The query feedback can be done across databases. For instance a reference from the Astronomy database can be used as query feedback in the preprint database to see the latest work in the field of this article.

If the article for the current reference has been scanned and is available through the ADS Article Service (see below), printing options are available in the abstract display as well. These printing options allow the printing of the article without having to retrieve the article in the viewer first.

\subsubsection{Article service}

This part of the ADS provides access to the scanned images of articles. We have received permission from most astronomy journals to scan their volumes and make them available on-line free of charge. A more detailed description of these data is in DATA.

The most common access to the scanned articles is through the ADS Abstract Service via the "G"-links (see above). However they can also be accessed directly through the article query page by publication year and month or by volume and page at:

\section{http://adsabs.harvard.edu/article_service.html}

This form returns the specified article in the user specified format (see Sect. 3). If a page within an article is specified, and the single page display is selected, the specified page within the article is returned with the links to the other available pages as usual.

The article display normally shows the first page (Fig. 7) of an article at the selected resolution and quality (see Sect. 3). The user can select resolutions of 75, 100, or 150 dots per inch (dpi) and image qualities of 1, 2, 3 , or 4 bits of greyscale per pixel. These gif images are produced on demand from the stored tiff images (see

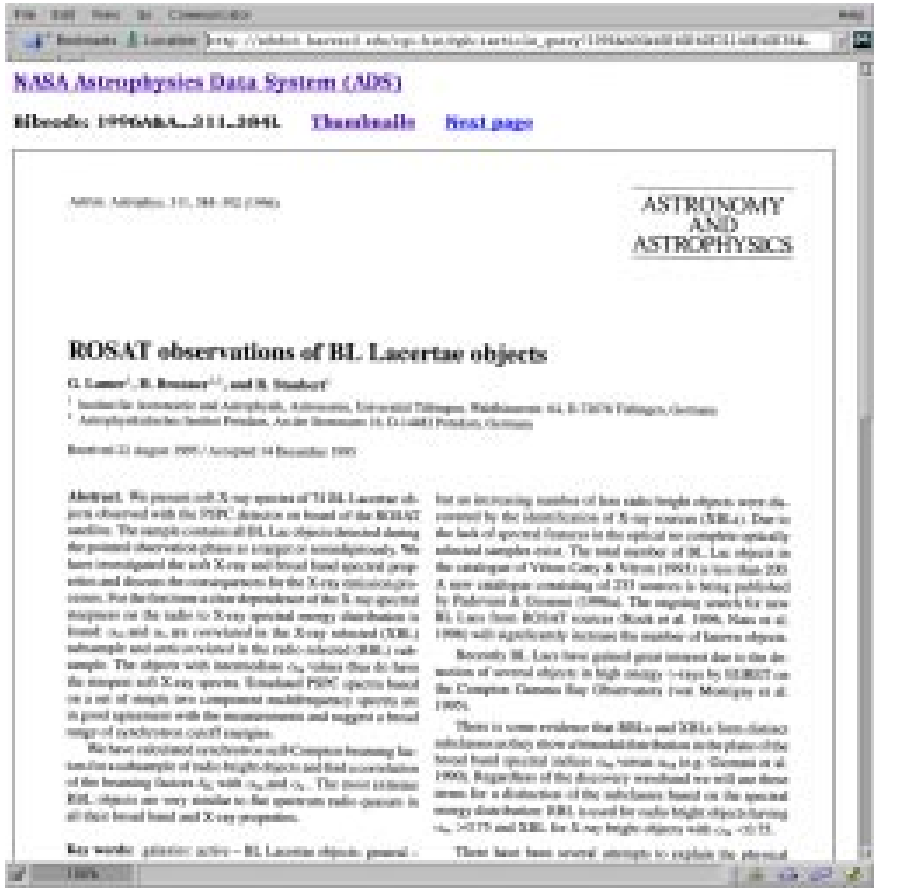

Fig. 7. The article display shows a gif image of the selected journal page with the resolution selected in the user preferences

DATA). The default version of the gif images (100 dpi, 3 bit greyscale) is cached on disk. The cache of these gif images is managed to stay below a maximum size. Any time the size of the cached gif images exceeds the preset cache size, the gif images of pages that have not been accessed recently are deleted.

Below the page image on the returned page are links to every page of the article individually. This allows the user to directly access any page in the article. Wherever possible, plates that have been printed separately in the back of the journal volume have been bundled with the articles to which they belong for ease of access. The next part of the displayed document provides access to plates in that volume if the plates for this journal are separate from the articles. Another link retrieves the abstract for this article.

The next part of the page allows the printing of the article. If the browser works with HTTP persistent cookies (see Sect. 3), there is just one print button in that section with a selection to print either the whole paper or individual pages. This print button will print the article in the format that the user has specified in the user preferences. If the browser does not handle cookies, several of the more commonly used print options are made available here.

All possible printing options can be accessed through the next link called "More Article Retrieval Options". This page allows the user to select all possible retrieval options. These include:

i. Postscript: Access to two resolutions is provided (200 dpi and $600 \mathrm{dpi}$ ). For compatibility with older 
printers, there is also an option to retrieve Postscript Level 1 files.

ii. PCL (Printer Control Language): This language is for printing on PCL printers such as the HP desk jets and compatibles.

iii. PDF (Portable Document Format): PDF can be viewed with the Adobe Acrobat reader (Adobe, Inc). From the Acrobat reader the article can be printed.

iv. TIFF (Tagged Image File Format): The original images can be retrieved for local storage. This would allow further processing like extraction of figures, or Optical Character Recognition (OCR) in order to translate the article into ASCII text.

v. Fax retrieval: Within the U.S.A., articles can be retrieved via fax at no cost. Again, the retrieval is greatly facilitated through the preferences setting capability. The preferences allow the user to store a fax number that will be used for the fax service.

vi. Email retrieval: Articles can be retrieved through email instead of through a WWW browser. MIME (Multipurpose Internet Mail Extension, Vaudreuil 1992) capable email systems should be able to send the retrieved images directly to the printer, to a file, or to a viewer, depending on what retrieval option was selected by the user.

For most of the retrieval options, the data can optionally be compressed before they are sent to the user. Unix compress and GNU gzip are supported compression algorithms.

Instead of displaying the first page of an article together with the other retrieval links, the user has the option (selected through the preferences system, see Sect. 3) to display thumbnails of all article pages simultaneously. This allows an overview of the whole article at once. One can easily find specific figures or sections within an article without having to download every page. This should be especially useful for users with slow connections to the Internet. Each thumbnail image ranges in size from only 700 bytes to 3000 bytes, depending on the user selected thumbnail image quality. The rest of this type of article page is the same as for the page-by-page display option.

\subsubsection{Other forms based user interfaces}

There are several forms available to directly access references or articles and other relevant information. All abstract query forms return the short reference format as described above. One form allows access to references through bibliographic codes:

http://adsabs.harvard.edu/bib_abs.html

This form allows the user to retrieve abstracts by specifying directly a bibliographic code or the individual parts of a bibliographic (year, journal, volume, page). This can be very useful in retrieving references from article reference lists, since such reference lists generally contain enough information to build the bibliographic codes for the references. This form also accepts partial codes and returns all references that match the partial code. It accepts the wildcard character "?". The "?" wildcard stands for one character in the code. For partial codes that are shorter than 19 characters, matching is done on the first part of the bibliographic codes. For instance:

1989ApJ...341?...1 will retrieve the articles on page 1 of the ApJ (Astrophysical Journal) and ApJ Letters volume 341 , regardless of the author name.

Another form allows access to the Tables of Contents (ToCs) of selected journals by month/year or volume:

http: //adsabs.harvard.edu/toc_service.html

One option on that form is to retrieve the latest published issue of a particular journal. Access to the last volumes of a set of journals is also available through a page with a graphical display of selected journals' cover pages:

\section{http: //adsabs . harvard . edu/tocs . html}

By clicking on a journal cover page either the last published volume of that journal or the last volume that the user has not yet read is returned, depending on the user preference settings (see Sect. 3). The information necessary for that service is stored with the user preferences in our internal user preferences database.

A customized ToC query page is available at:

http://adsabs.harvard.edu/custom_toc.html

It will display only icons for journals that have issues available that have not been read by the user. This allows a user to see at a glance which new issues for this set of journals have been published. The set of journals that is included in the customized ToC query page can be specified in the user preferences (see Sect. 3).

As mentioned in Sect. 2.1.1 and in ARCHITECTURE, one important aspect of the ADS search system is the list of synonyms. Sometimes it is important for our users to be able to see what words are in a particular synonym group to properly interpret the search results. Another question that is asked is what words are in the database and how often. The list query page (linked to the words "Authors", "Title Words", and "Text Words" above the corresponding entry fields on the main query form) allows the user to find synonym groups and words in the database. The user can specify either a complete word in order to find its synonyms, or a partial word with wildcard characters to find all matching words in the database. When a word without wildcard characters is specified, the list query form returns all of its synonyms (if any).

To find words matching a given pattern, the users can specify a partial word with either or both of two wildcard characters. The question mark "?" stands for any single character, the asterisk "*" stands for zero or more characters (see Sect. 4.2.3). For a wildcard search, the list query 
form returns all words in the database that match the specified pattern, together with the frequencies of these words in the database.

\subsubsection{Journal specific access forms}

The regular query forms search the complete database. The user can select the return of only specific journals in the "Filter" section of the query form. In order to allow different journals to use the ADS system for searching their references, journal specific pages are available. The URL (Uniform Resource Locator) for an abstract search page for a specific journal is:

http: //adsabs.harvard.edu/Journals / search/bibstem

The page for retrieving scanned articles of a specific journal is:

http: //adsabs.harvard.edu/Journals/ articles/bibstem

and the page for retrieving the tables of contents by volume or publication date is:

http://adsabs.harvard. edu/Journals/toc/bibstem

In each case, bibstem is the abbreviation for the selected journal. For instance:

http: //adsabs . harvard.edu/Journals/search/ApJ

returns a query form for searching only references of the Astrophysical Journal. These forms are available for linking by anybody.

\subsection{Direct access interfaces}

Both abstracts and articles can be accessed directly though HTML hyperlinks. The references are identified through the bibliographic codes (or bibcodes for short) mentioned above and described in detail in DATA. The syntax for such links to access abstracts is:

http://adsabs.harvard.edu/cgi-bin/ bib_query?bibcode=1989ApJ . . .342L . .71R

Scanned articles can be accessed directly through links of the form:

http://adsabs.harvard.edu/cgi-bin/ article_query?bibcode=1989ApJ . . .342L . .71R

These links will return the abstract or scanned article respectively for the specified bibliographic code. They are guaranteed to work in this form. You may see other URLs while you use the ADS. These are internal addresses that are not guaranteed to work in the future. They may change names or parameters. Please use only links of the form described above to directly access abstracts and articles.

\subsection{Embedded queries}

Embedded queries can be used to build hyperlinks that return the results of a pre-formulated query. One frequently used example is a link that returns all articles written by a specific user. The syntax for such a link is:

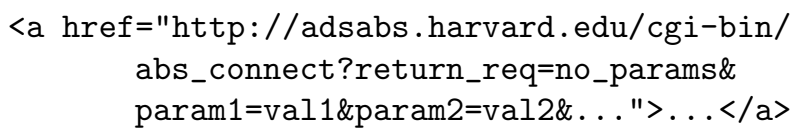

There are no spaces allowed in a URL. All blanks need to be encoded as "+". The parameter return_req=no_params sets all the default settings. Individual settings can be changed by including the name of the specific setting and its value after the return_req=no_params parameter in the URL. A list of available parameters can be accessed at:

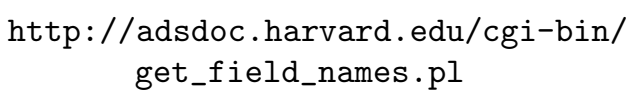

We try to make changes to parameters backward compatible, but that is not always possible. We encourage you to use this capability, but it is advisable to use only the more basic parameters.

This type of interface allows users to link to the ADS for a comprehensive list of references on a specific topic. Many users use this to provide an up-to-date publication list for themselves by encoding an author query into an embedded query.

\subsection{Perl script queries}

The ADS database can be used by other systems to include ADS data in documents returned from that site. This allows programmers at other sites to dynamically include the latest available information in their pages. An example is the interface that SPIE (the International Society for Optical Engineering) provides to our database. It is available at:

http://www.spie.org/incite/

This site uses Perl scripts to query our database and format the results according to their conventions. These Perl scripts are available at:

http: //adsabs.harvard. edu/www/adswww-lib/

The Perl scripts allow the programmer to specify all the regular parameters. The results are returned in Perl arrays. If you use these scripts, we would appreciate it if you would credit the ADS somewhere on your pages. 


\subsection{Email interface}

The ADS Abstract Service can be accessed through an email interface. This service is somewhat difficult to use since it involves an interface between two relatively incompatible interface paradigms. This makes describing it quite difficult as well. It is intended for users who do not have access to web browsers. If you have questions about how to use this access, please contact the ADS at ads@cfa.harvard.edu.

To get information about this capability, send email to:

adsquery@cfa.harvard.edu

with the word "help" in the message body.

This interface accepts email messages with commands in the message body. The subject line is ignored. The commands that are available are:

1) help (see above)

2) action=URL

The second command allows a user to retrieve a document at the specified URL. Three qualifiers allow the user to specify what retrieval method to use, what format to return, and to which address to return the results:

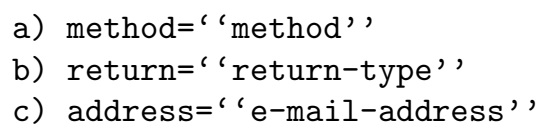

a. "method" is either "get" or "post" (without the quotes). This determines what kind of query will be executed. To retrieve a form for further queries, use the 'get' method. To execute a forms query you need to know what type of query the server can handle. If you execute a forms query after retrieving the form through this service, the correct method line will already be in place. Default method is "get".

b. "return-type" is either "text", "form", or "raw" (without the quotes). If text return is requested, only the text of the query result is returned, formatted as if viewed by a WWW browser. If form return is requested, the text of the result is returned as well as a template of the form that can again be executed with this service. If raw return is requested, the original document is returned without any processing. Default return is "form". The capability to return MIME encoded files is in preparation.

c. "e-mail-address" specifies to which e-mail address the result should be sent. This line is optional. If no address is specified, the result is sent to the address from where the request came.

To execute a query via email, the user first retrieves the abstract query form with:

\section{action=http: //adsabs.harvard.edu/ abstract_service.html}

This will return an executable form. This form can be returned to the ADS in an email message to execute the query. The user enters input for the different fields as
Table 2. Database names supported in the ADS database

\begin{tabular}{|c|c|}
\hline Value & Description \\
\hline $\mathrm{AST}$ & $\begin{array}{l}\text { Astronomy Database. Contains references from } \\
\text { Astronomy related articles }\end{array}$ \\
\hline INST & $\begin{array}{l}\text { Instrumentation Database. Contains references } \\
\text { from articles related to Space Instrumentation }\end{array}$ \\
\hline PHY & $\begin{array}{l}\text { Physics Database. Contains references from } \\
\text { Physics related articles }\end{array}$ \\
\hline PRE & $\begin{array}{l}\text { Preprint Database. Contains references from the } \\
\text { Los Alamos preprint server related to Astronomy }\end{array}$ \\
\hline GEN & $\begin{array}{l}\text { General Database. Contains references from } \\
\text { articles unrelated to the other databases }\end{array}$ \\
\hline
\end{tabular}

Table 3. Use attributes supported in the ADS database

\begin{tabular}{ll}
\hline Value & Description \\
\hline 1 & Personal name $^{\mathrm{a}}$ \\
1003 & Author $^{\mathrm{a}}$ \\
4 & Title \\
5 & Title series $^{\mathrm{b}}$ \\
62 & Abstract \\
31 & Publication Date \\
1011 & Entry Date in Database \\
\hline
\end{tabular}

a These attributes search the same field.

b This attribute limits searches to the journal specified.

required in the forms template. For forms tags like checkboxes or radio buttons, the user can uncomment the appropriate line in the form. Comments in the form that are included with each forms field provide guidance for completing the form before submission. The text part of the form is formatted as comment lines so that the user does not have to modify any irrelevant parts of the form. The retrieval method is already set appropriately.

\subsection{Z39.50 interface}

The recently implemented Z39.50 interface (Lynch 1997) conforms to the Library of Congress Z39.50 conventions (http://lcweb.loc.gov/z3950/lcserver.html). This allows any library that uses this protocol to access the ADS through their interface. The databases supported through this interface are listed in Table 2, search fields supported are listed in Table 3, supported relationship attributes are listed in Table 4 and the supported structure attributes are listed in Table 5. Table 6 lists the supported record formats and Table 7 shows the supported record syntax.

Each table notes which search fields support which attribute. The relationship attributes "equal" and "relevance" are used to search without and with synonym replacement respectively. If the structure attribute "Phrase" is selected, the input is considered to be one phrase and 
Table 4. Relation attributes supported in the ADS database

\begin{tabular}{lll}
\hline Value & Description & Fields Supporting \\
\hline 3 & Equal $^{\mathrm{a}}$ & All \\
2 & Less than or Equal & Publication Date \\
4 & Greater than or Equal & Publication Date, Entry \\
& Date & \\
102 & Relevance $^{\mathrm{b}}$ & Title, Abstract, Author \\
\hline
\end{tabular}

${ }^{a}$ Relation Equal searches for exact words.

b Relation Relevance searches for words and their synonyms.

Table 5. Structure attributes supported in the ADS database

\begin{tabular}{lll}
\hline Value & Description & Fields Supporting \\
\hline 1 & quoted phrase & Title, Abstract \\
2 & word & Title, Abstract, Author, Series \\
6 & word list & Title, Abstract, Author, Series \\
5 & date & Publication Date, Entry Date \\
\hline
\end{tabular}

Table 6. Record format supported in the ADS database

\begin{tabular}{ll}
\hline Value & Description \\
\hline B & Brief Records (Title, Authors) \\
F & Full Records (all available information) \\
\hline
\end{tabular}

Table 7. Record syntax supported in the ADS database

\begin{tabular}{ll}
\hline Value & Description \\
\hline 1.2 .840 .10003 .5 .101 & SUTRS Records \\
1.2 .840 .10003 .5 .109 .3 & HTML Records \\
1.2 .840 .10003 .5 .1000 .147 .1 & ADS Tagged Records \\
\hline
\end{tabular}

is not separated into individual words. If the structure attribute "Word" is selected and several words are specified, the input is treated as if the structure attribute "Word List" were specified.

As output, brief and full records are supported. These record formats are the same as in the short results list and in the full abstract display as described above. The supported record syntax is either SUTRS (Simple Unstructured Text Record Syntax), HTML (HyperText Markup Language), or the ADS tagged format. In the HTML record syntax, links to other supported ADS internal and external data sources are included.

A description of this interface is available on-line at:

http://adsabs.harvard.edu/abs_doc/ads_server.html

An example of the ADS Z39.50 interface can be accessed from the Library of Congress Z39.50 Gateway at:

http://lcweb.1oc.gov/z3950/

\section{Preferences}

The ADS user interface is customized through the use of so-called HTTP persistent cookies (see Kristol \& Montulli 1997). These "cookies" are a means of identifying individual users. They are strings that are created by the server software. Web browsers that accept cookies store these identification strings locally on the user's computer. Anytime a user makes a request, the ADS software checks whether the requesting browser is capable of accepting cookies. If so, it sends a unique string to the browser and asks it to store this string as an identifier for that user. From then on, every time the same user accesses the ADS from that account, the browser sends this cookie back to the ADS server. The ADS software contains a database with a data structure for each cookie that the ADS has issued. The data structure associated with each cookie contains information such as the type of display the user prefers, whether tables should be used to format data, which mirror sites the user prefers for certain external data providers, the preferred printing format for ADS articles, and which journal volumes the user has read. It also can store the email address of the user and a fax number, in case the user wants to retrieve articles via email or fax.

The preference settings form includes a field for the user name as well as the email address. However, neither is necessary for the functioning of any of the features of the ADS. The system is completely anonymous. None of the information stored through this cookie system is made available to anybody outside the ADS. There is no open interface to this database and the database files are not accessible through the WWW. Any particular user can only access their own preferences, not the preferences set by any other user.

Most of these preferences can be set by the user through a WWW forms interface. Some fields in a user's preference record are for ADS internal use only. For instance the system is being used to display announcements to users in a pop-up window. The cookie database remembers when the message was last displayed, so that each message is displayed only once to each user.

This preference saving system also allows each user to store a query form with filled-in fields. This enables users to quickly query the ADS for a frequently used set of criteria.

The cookie identification system is implemented as a Berkeley DBM (DataBase Manager) database with the cookie as the record key. The data block that is stored in the database is a $\mathrm{C}$ structure. The binary settings (e.g. "Use Tables", or "Use graphical ToC Page") are stored as bits in several preference bytes. Other preferences are stored as bytes, short integer, or long integer, depending on their dynamic range. 


\section{Search engine details}

\subsection{General}

The search engine software is written in C. It accepts as input a structure that contains all the search fields and flags for the user specified settings and filters. For each search field that contains user input a separate POSIX (Portable Operating System Interface) thread is started that searches the database for the terms specified in that field. The results obtained for each term in that field are combined in the thread according to the specified combination logic. The resulting list of references is returned to the main thread. The main thread combines the results from the different field searches and calculates the final score for each reference. The final combined list with the scores is returned to the user interface routines that format the results according to the user specified output format.

\subsection{Searching}

\subsubsection{Database files}

The abstracts are indexed in separate fields: Author names, titles, abstract text, and objects. Each of these fields is indexed similarly into an index file and a list file (see ARCHITECTURE). The index file contains a list of all terms in the field together with the frequency of the term in the database, and the position and length of two blocks of data in the list file. One block contains all references that include the exact word as specified. The other block contains all references that contain either the word or any of its synonyms.

A search for a particular word in the index file is done through a binary search in the index. The indexes are resident in memory, loaded during boot time (see Sect. 5). Once the word is found in the index, the position and length of the data block is used to directly access the data block in the list file. This data block contains the identifier for each reference that contains the search word.

If a quick update has occurred (see ARCHITECTURE) since the list file was last built (indicated by a negative in part of the last identifier), an auxiliary block of reference identifiers is read. Its position is contained in the structure of the last reference identifier. This auxiliary block is merged with the original one.

The identifier for each reference is the position of the bibliographic code for that reference in the list of all bibliographic codes. This system saves one lookup of the identifier in a list of identifiers, since the number can be used directly as an index in the array of bibliographic codes.

\subsubsection{Synonym searches}

As mentioned above, the index files contain information about two blocks of data, the data for the individual word and for the synonym group to which this word belongs. When a search with synonym lookup enabled is requested, the block of data for the whole synonym group is used, otherwise the data for only the individual word is returned. All the processing that enables these two types of searches is done during indexing time, therefore the speeds for both searches are similar.

Even though our synonym list is quite extensive (see ARCHITECTURE) our users will sometimes use words that are not in the database or the synonym list. In these cases the search software uses a stemming algorithm from the Unix utility ispell to find the stem of the search word and then searches for the word stem. The indexing software has indexed the stems of all words in the database together with their original words (see ARCHITECTURE). This word stemming is done as a last resort if no regular match has been found in an attempt to find any relevant references.

\subsubsection{Wildcard searches}

In order to be able to search for families of words, a limited wildcard capability is available. Two wildcard characters are defined: The question mark "?" is used to specify a single wildcard character and the asterisk "*" is used to specify zero or more wildcard characters. The "?" can be used anywhere in a word. For instance a search for $M 1$ ? will find all Messier objects between M 10 and M 19. A search for $a$ ? sorb will find references with absorb as well as adsorb.

The asterisk can only be used at the beginning or at the end of a word. For instance $3 C^{*}$ searches for all $3 \mathrm{C}$ objects. * sorb searches for words that end in sorb like absorb, desorb, etc. When synonym replacement is on, all their synonyms (e.g. absorption) will be found as well. The "?" and the "*" can be combined in the same search string.

\subsection{Results combining within a field}

\subsubsection{Combining results}

As mentioned above, the user can select between four types of combination methods: "OR", "AND", simple logic, and full boolean logic. For the first three cases, the references for all search terms are retrieved and sorted first. The reference lists are then merged by going through the sorted reference lists sequentially and synchronously and selecting references according to the chosen logic.

The search algorithm for the full boolean logic is different. The boolean query is parsed from left to right. For 


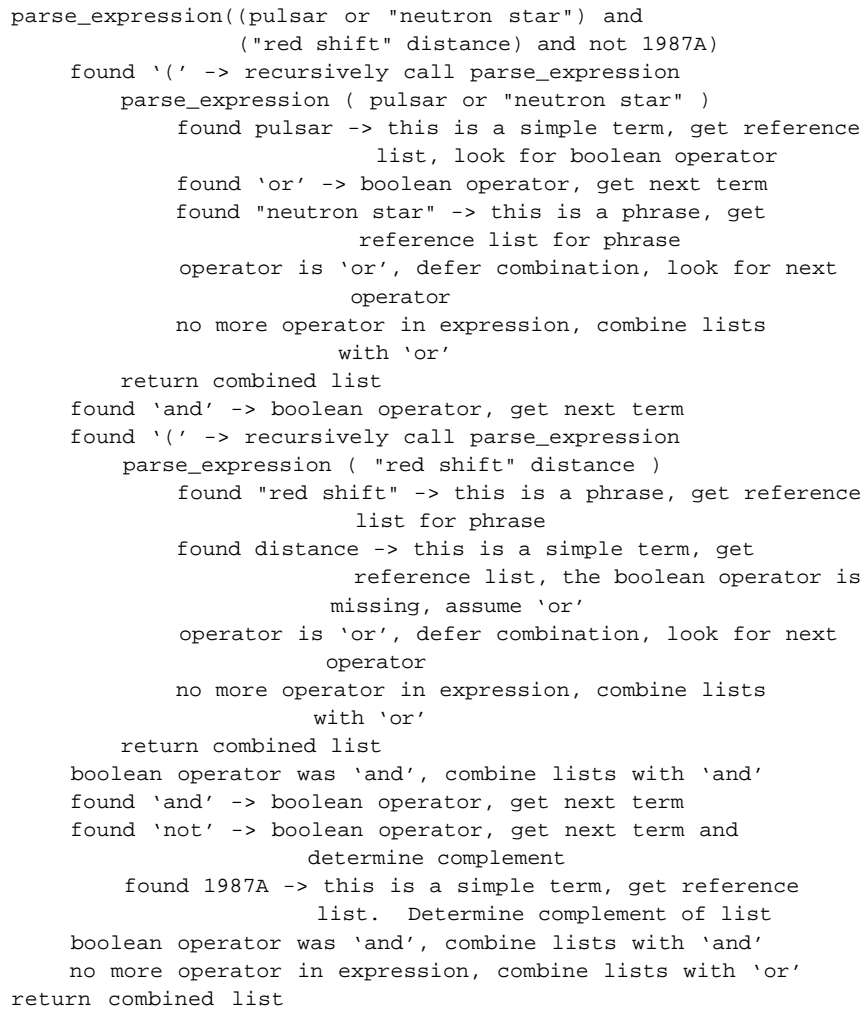

Fig. 8. Pseudo code for parsing full boolean search expression

each search term a function is called that finds the references for this term. A search term is either a search word, a phrase, or an expression enclosed in parentheses. If the search term contains other terms (if it is enclosed in parentheses), the parsing function is called recursively.

The next step is to determine the boolean operator that follows the search term, and then to evaluate the next search term after the operator. Once the reference lists for the two terms and the combining operator are determined, the two lists are combined according to the operator. This new list is then used as the first term of the next expression.

If the boolean operator is "OR", the combining of the lists is deferred, and the next operator and search term are evaluated. This ensures the correct precedence of "OR" and "AND" operators.

The "NOT" operator is implemented by getting the reference list for the term, making a copy of all references in the database, and then removing the references from the search term from the complete list. This yields a very large list of references. If the first search term in a boolean expression is a "NOT" term, the search will take very long, because this large list has to be propagated through all the subsequent parsing of the boolean expression. Care should therefore be taken to put a "NOT" term to the right of at least one other term, since processing is done left to right.

As an example, Fig. 8 shows the processing of the boolean expression mentioned in Sect. 2:

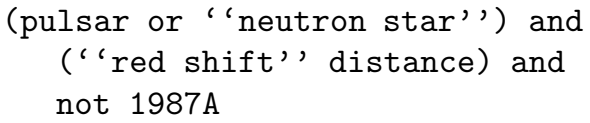

\subsubsection{Scoring}

In addition to the information about the references for each word, the index file also contains its frequency in the database. The frequency is already pre-calculated as $\operatorname{int}(10000 /(\log ($ frequency $)))$ during indexing (see ARCHITECTURE). This saves considerable time during execution of the search engine since all server calculations can be done as integer computations, no floating point operations are necessary. During the first part of the search, this frequency is attached to each retrieved reference. In the next step, the retrieved references are combined according to the selected combination logic for that field.

For "OR" combination logic, the lists retrieved for each word are merged and uniqued. As described in Sect. 5, this is done by going through the sorted reference lists synchronously and adding each new reference to the output list. The score for that reference is determined by adding up the frequencies from each of the lists for weighted scoring, or by setting a score equal to the number of matched words for proportional scoring.

For "AND" combination logic, only references that appear in every one of the lists are selected. Each of these references receives a score of 1 .

For simple logic and full boolean logic, the score for the returned references is determined only from the search terms that were combined with "OR". All words that have mandatory selection criteria (prefixed by "+" or "_" in simple logic, and combined with "AND" or "NOT" in full boolean logic) do not affect the final score.

\subsection{Combining results among fields}

\subsubsection{Combining}

After the POSIX threads for each search field are started, the main program waits for all threads to complete the search. When all searches are completed the search engine combines the results of the different searches according to the selected settings. If for instance one field was selected as required for selection in the settings section of the query form, only references that were found in the search for that field will be in the final reference list. The combined list is then uniqued and sorted by score. The resulting list of references is passed back to the user interface software.

If the user did not specify any search terms, a date range has to be selected. The software queries the database for all references in the selected date range and uses this list for further processing, e.g. filtering (see Sect. 4.5). 


\subsubsection{Scoring}

The score for each reference in the final results list is determined by adding the scores from each list multiplied by the user specified weight for each field and then normalizing the score such that a reference that matches all search terms from all fields receives a score of 1 .

\subsection{Selection filters}

After the search is completed according to the specified search words and the settings that control the combination logic and the scoring algorithms, the resulting list of references can be filtered according to several criteria. During the design of the software a decision had to be made whether to filter the results while selecting the references or after completing the search. The first approach has the advantage that the combining of the selected references will be faster because fewer references need to be combined. The second approach has the advantage that the first selection is faster. We chose the second approach because, except for selecting by publication date, only a small number of queries use filtering (see Sect. 6). Because of that, filtering by publication date is done during selection of the references, while the other filtering is done after the search is completed.

References can be filtered by five criteria:

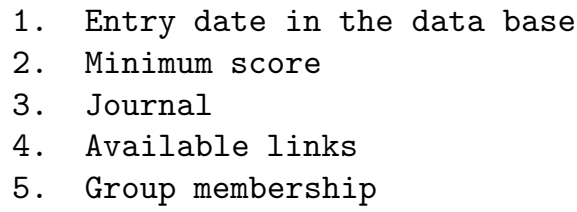

1. +2 . Entry date and minimum score. These two filters can be used to query the database automatically on a regular basis for new information that is relevant to a selected topic. The user can build a query form that returns relevant references, and then save this query form locally. This query form can then be sent to the ADS email interface (see above) on a weekly or monthly basis. By specifying an entry day of -7 for instance, the query will retrieve all references that fit the query and that were entered within the last seven days. The minimum score can be used to limit the returned number of references to only the ones that are really relevant. The references are returned via email as described in Sect. 2.5 about the email interface.

3. Journal filter. This filter allows the user to select references from individual journals or groups of journals. Available options for this filter are:

a. All journals (default)

b. Refereed journals only

c. Non-refereed journals only

d. Selected journals
If the last option is selected, the user can specify one or more journal abbreviations (e.g. ApJ, AJ (Astronomical Journal)) that should be selected. More than one abbreviation can be specified by separating them with semicolons or blanks. The filter for journals can also include the volume number (but not the page number). The journal abbreviation is compared with the bibliographic codes over the length of the specified abbreviation. For instance if the user specifies $A p J$, the system selects all articles published in the ApJ, ApJ Supplement and ApJ Letters. ApJ.. will select only articles from the ApJ and the ApJ Letters. A special abbreviation, $A p J L$ will select only articles from the ApJ Letters. If a journal abbreviation is specified with a prepended "-", all references that are NOT from that journal are returned. The journal abbreviations (or bibstems) used in the ADS are available at:

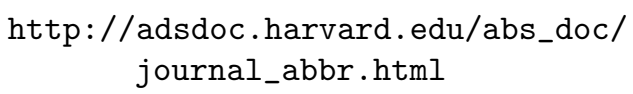

4. Available links. This filter allows the user to select references that have specific other information available. The returned references can be filtered for instance to include only references that have data links or scanned articles available. As an example, a user needs to find on-line data about a particular object. A search for that object in the object field and a filter for references with on-line data returns all articles about that object that link to on-line data.

5. Groups. We provide the capability to build a reference collection for a specific subset of references. This can be either articles written by members of a particular research institute or about a particular subject. Currently there are 5 groups in the ADS. We encourage larger institutes or groups to compile a list of their references and send it to us to be included in the list of groups.

\section{Optimizations}

The search engine is entirely custom-built software. As mentioned in the introduction, the first version of the Abstract Service used commercial database software. Because of too many restrictions and serious performance problems, a custom-designed system was developed. The main design goal was to make the search engine as fast as possible. The most important feature that helped speed up the system was the use of permanent shared memory segments for the search index tables. In order to make searching fast, these index tables need to be in Random Access Memory. Since they are tens of megabytes long, they cannot be loaded for each search. The use of permanent shared memory segments allows the system to have all the index tables in memory all the time. They are loaded during system boot. When a search engine is started, it attaches to the shared segments and has the data available immediately without any loading 
delays. The shared segments are attached as read-only, so even if the search engine has serious bugs, it cannot compromise the integrity of the shared segments. Using shared segments with the custom-built software improved the speed of a search by a factor of $2-20$, depending on the type of search.

Access to the list files (see Sect. 4.2) was optimized too. These files cannot be loaded into memory since they are too large (each is over one hundred megabytes in size). To optimize access to these files, they are memory mapped when they are accessed for the first time. From then on they can be accessed as if they were arrays in memory. The data blocks specified in the index tables can be accessed directly. Access is still from file, but it is handled through the paging in the operating system, rather than through the regular I/O system, which is much more efficient.

Once the search engine was completed and worked as designed, it was further optimized by profiling the complete search engine and then optimizing the modules that used significant amounts of time. Further analysis of the performance of the search engine revealed instances where operations were done for each search that could be done during indexing of the data and during loading of the shared segments. Overall these optimizations resulted in speed improvements of a factor of more than 10 over the performance of the first custom-built version. These optimizations were crucial for the acceptance of the ADS search system by the users.

In order to further speed up the execution, the search engine uses POSIX threads to exploit the inherent parallel nature of the search task. The search for each field, and in the case of the object field for each database, is handled by a separate POSIX thread. These threads execute in parallel, which can provide speedups in our multiprocessor server. Even for single processor systems this will provide a decrease in search time, since each thread sometimes during its execution needs to wait for I/O to complete. During these times other threads can execute and therefore decrease the overall execution time of a search.

Another important part of the optimization was the decision on how to structure the index and list files. The index files contain the word frequency information that is used to calculate scores for the weighted scoring (see Sect. 2.1.1c). The score for a matching reference is calculated from the inverse logarithm of the frequency of the word in the database. This requires time consuming floating point calculations. To avoid these calculations during the searches, the floating point arithmetic is done at indexing time. The index file contains the inverse log of the word frequency multiplied by a normalization factor of 10 000. This allows all subsequent calculations to be done in integer arithmetic, which is considerably faster than floating point calculations.

Another optimization was to pre-compile the translation rules (see Sect. 2.1.1). These translation rules are pre-compiled and stored in a shared memory segment to which the search process attaches. This allows for faster execution of these pattern matching routines.

Overall, these optimizations improved the speed of the searches by two orders of magnitude between the original design using a commercial database and the current software.

\section{Access statistics}

The ADS software keeps extensive logs about the use of the search and access software. In this section, usage statistics for the search software and for access patterns to the Article Service are reported. If not otherwise indicated, the statistics in this section are for the one-year period from 1 April 1998 through 31 Mar. 1999.

\subsection{Abstract service}

The ADS is accessed by users from many different countries. In the one-year period of this section the ADS was accessed by 127000 different users, using 100000 different hosts from 112 different countries. An individual user is defined as having a unique cookie (see Sect. 3). Users without cookies are distinguished by the hostnames from which the requests came. This may overestimate the number of users, since some users may have more than one cookie, for instance when accessing the ADS from home. The number of different hosts is a lower limit of the number of users. Many hosts are used by multiple users, so the real number is certainly considerably higher than that. The development of the number of users and queries over the life of the ADS is described in OVERVIEW. This section describes some more detailed investigations of the access statistics.

The total number of users at first comes as quite a surprise. The number of working astronomers in the world is probably between 10000 and 20 000. The number of ADS users is much larger than that. This is probably due to several factors. First, there are certainly many accidental users. They somehow find our search page through some link, execute a query to see what they get back, and then never come back because it is of no interest to them.

Other possible users are media people. There are certainly many reporters occasionally looking up something in astronomy. I have spoken with several of them that use the ADS occasionally for that.

Another group of users are amateur astronomers. The ADS was described in Sky \& Telescope by Eichhorn (1996a) a few years ago. this has certainly made amateur astronomers aware of this resource. The number of amateur astronomers world-wide is certainly in the millions, so they comprise a potentially large number of users.

Another large group of users visits the ADS through links from other web sites. One particularly popular one is 


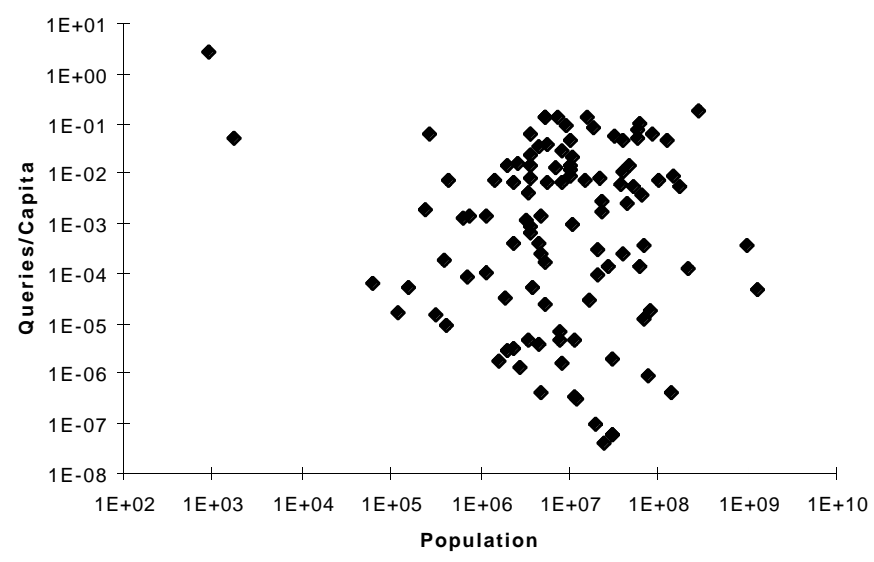

Fig. 9. Number of queries per person per year in each country as a function of the population for the country

NASA's Image of the Day, which frequently includes links to abstracts or articles in the ADS. Since this NASA page is visited by millions of people, a large number of them will access the ADS through these links.

The use of the ADS in different countries depends on several factors. One of these is certainly the population of the country. Figure 9 shows the number of queries per capita as a function of the population of the country (CIA 1999). There seems to be an upper limit of about $0.1-0.2$ queries per person per year. The one exception is the Vatican with almost 3 queries per person per year. This is understandable since the Vatican has an active Astronomy program, which generates a large number of queries for a small population.

Another factor for querying the ADS is the funding available for Astronomy in a country, and the available infrastructure to do astronomical research. Figure 10 shows the number of references retrieved per capita as a function of the Gross Domestic Product (GDP, CIA 1999) of the country. The symbols are the Internet codes for each country.

The highly industrialized countries cluster in the upper right part of the plot (area 1). A closeup of this region is shown in Fig. 11. Other clusters are the countries of the former Soviet Union (area 2), and Central and South American countries (area 3). The high number of references retrieved per capita combined with the lower GDP per capita of the former Soviet Union is probably due to a recent decline in GDP, but a still existing infrastructure for astronomical research.

The ADS is used 24 hours per day. The distribution of queries throughout the day is shown in Fig. 12. This figure shows the number of queries at the two largest mirror sites, as well as the queries at the main ADS site. The usage distribution data are for the time period from 1 November 1998 to 31 March 1999, not the full year, to avoid complications due to different periods where daylight savings time is in effect. The queries at the main site are separated into US users and non-US users on the basis

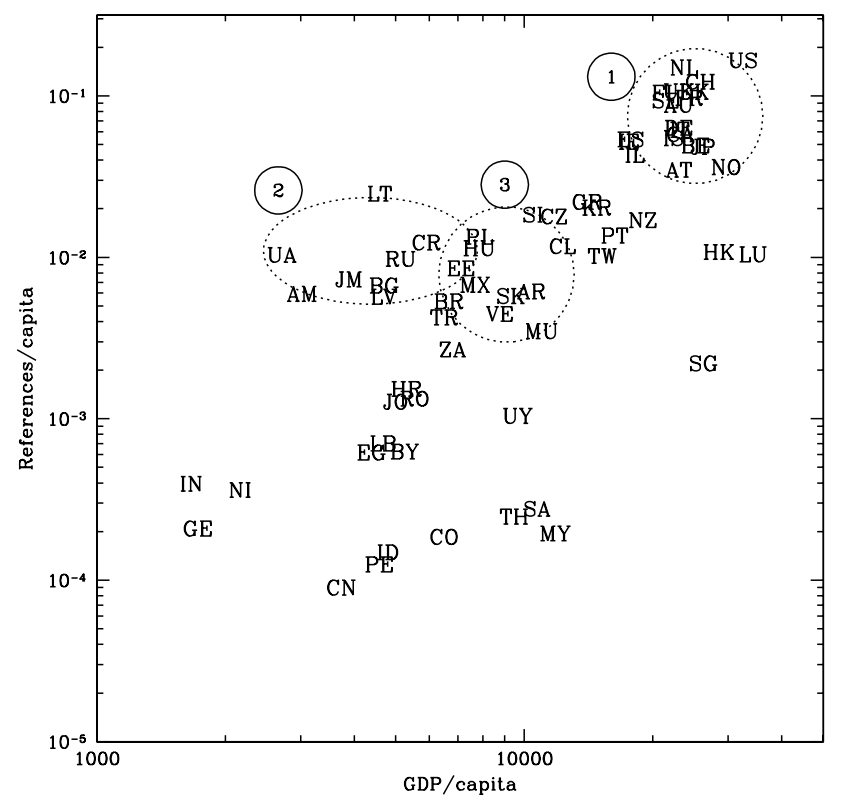

Fig. 10. Number of references retrieved per person as a function of the Gross Domestic Product (GDP) per person for each country

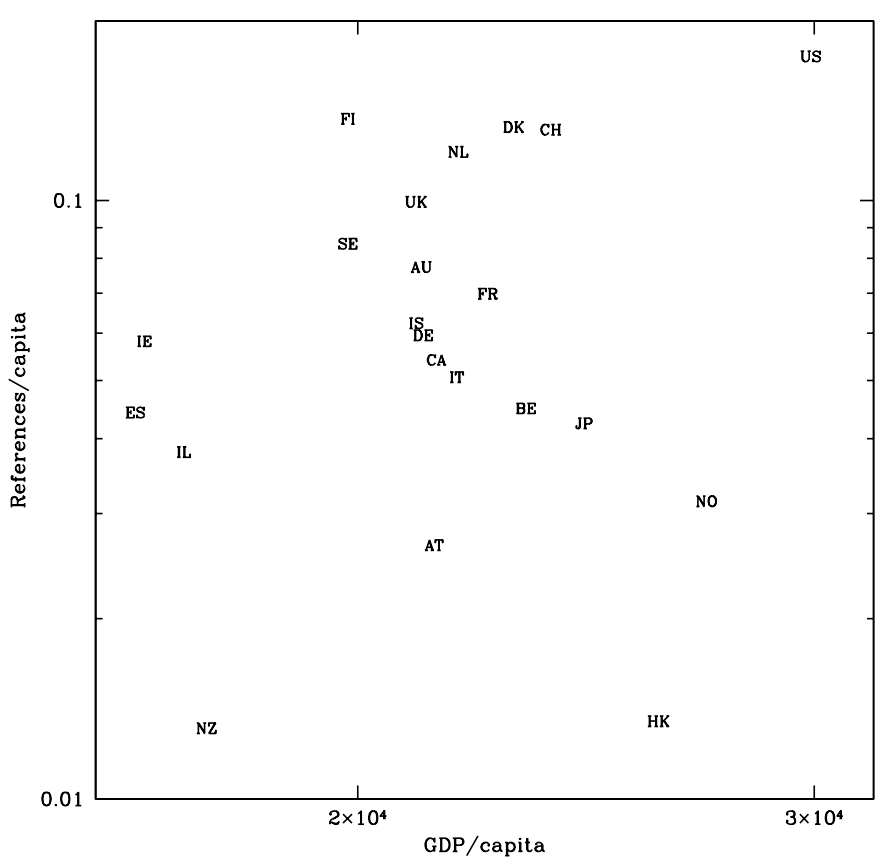

Fig. 11. Number of references retrieved per person as a function of the Gross Domestic Product (GDP) per person. This figure is a closeup of area 1 in Fig. 10 


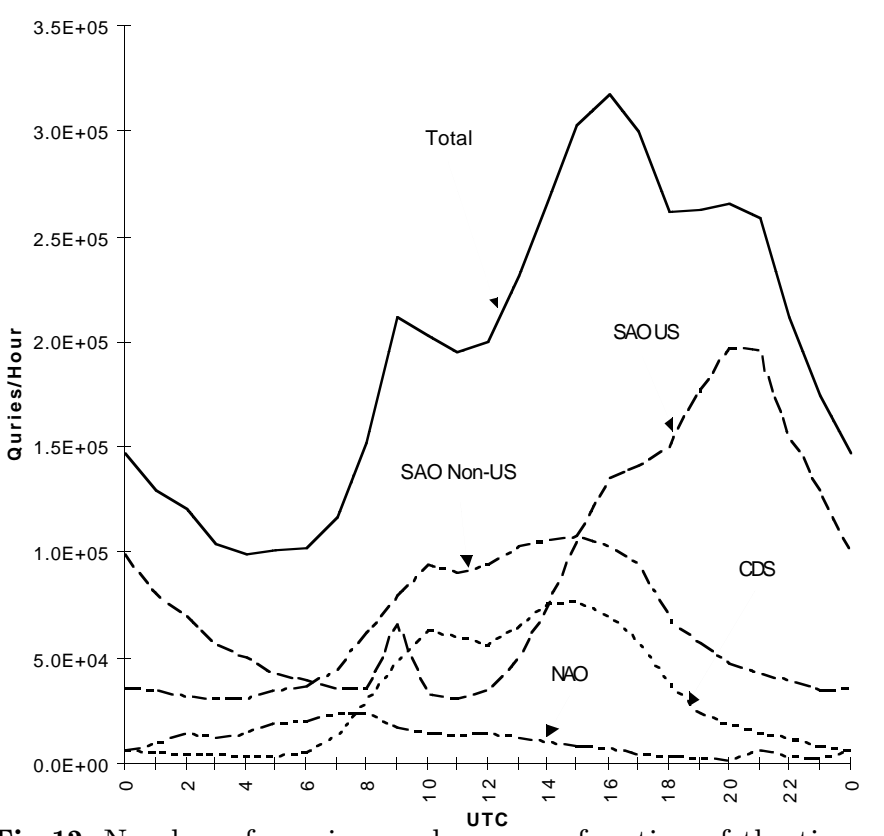

Fig. 12. Number of queries per hour as a function of the time of day for the main SAO site and the mirror sites in France and Japan

of their Internet hostnames. All the individual curves show a distinct two-peaked basic shape, with additional smaller peaks in some cases. This distribution of queries over the day shows the usage throughout a workday, with a small minimum during lunch hour. The SAO-US distribution does not show a real minimum between the two peaks, presumably because of the distribution of US researchers over three time zones.

There are three features in this figure that deserve special notice. The first is that the shape of the accesses to the ADS mirror in France is the same as the shape of the non-US access to the SAO site. This indicates that the large majority of the non-US use on the SAO site is from European users.

This non-US usage is about $50 \%$ higher than the total usage of the ADS mirror site at the CDS in France. The reason for this is most probably the fact that the connectivity within Europe is not yet very good. We know for instance that our users in England and Sweden have better access to the main ADS site in the U.S.A. than to our mirror site in France. The same is true for other parts of Europe.

Another reason for the use of the U.S.A. site by European users is the fact that our European mirror sites do not yet have the complete set of scanned articles online. This forces some users to access the main ADS site in order to retrieve scanned articles.

Second, there is a slight peak in the distribution of queries to the NAO mirror in Japan around 21:00 UTC (Universal Time Coordinated, formerly Greenwich Mean Time). This is probably due to US west coast users using the Japanese mirror site instead of the US site. The access

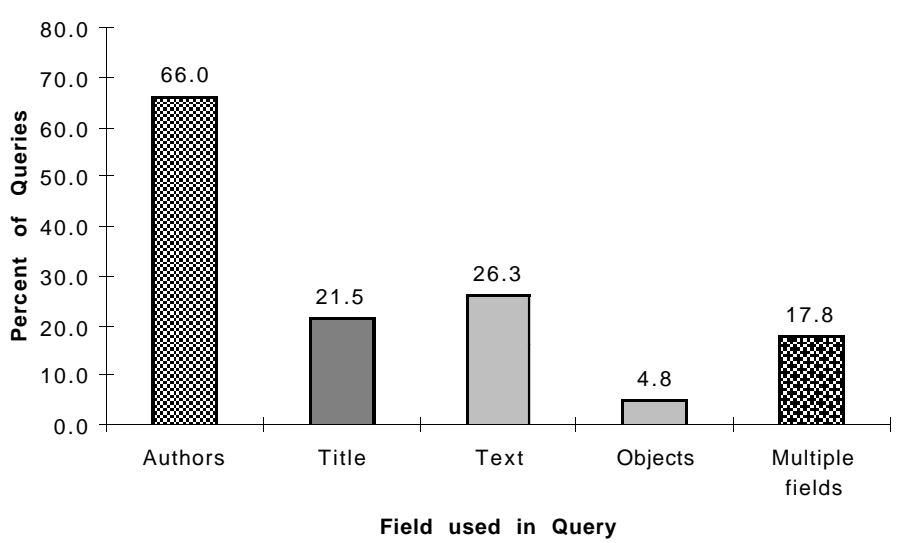

Fig. 13. A histogram of the relative usage of the different search fields (authors, objects, title, text) and the use of multiple fields

to Japan is frequently very fast and response times from Japan may be better than from SAO during peak traffic times.

Third, there is a distinct peak in the SAO-US usage at 9:00 UTC. This feature was so unusual that we tracked down the reason for it. It turns out that one of our users has set up web pages that include about 200 links to ADS abstracts. He had set up a link verifier that every night at 9:00 UTC checked all the links on his pages. This meant that the link verifier executed 200 queries every night at the same time, which showed up in this evaluation of our access statistics.

The following section shows statistics of how our users use the different capabilities of the ADS query system. Figure 13 shows a histogram of the relative usage of the different search fields (authors, objects, title, text). It shows clearly that the majority of queries are queries by author name $(66 \%)$. Object names are used in fewer than $5 \%$ of the queries. The title field is used in about $21 \%$ of the queries, and the text field in 26\%. Queries that use more than one field make up about $18 \%$ of the total. This usage pattern justifies for instance including tables of contents (ToCs) in the database that do not have abstracts for searching. Since a large part of the usage is through author and title queries, such ToC entries will still be found.

Figure 14 shows the number of queries as a function of the number of query items in each input field. The query frequency generally decreases exponentially with increasing number of search terms. For title and text queries, the frequency is approximately constant up to 3 query words, before the frequency starts to decrease. For abstract queries there is a significant increase in frequency of queries with more than 20 query words, for title queries there is a similar increase for queries with more than about 8 query words. This is due to queries generated through the query feedback mechanism which allows the user to use a given abstract and its title as a new query.

Figures 15 and 16 show the usage of non-default query settings (see Sect. 4.2). The default settings were 


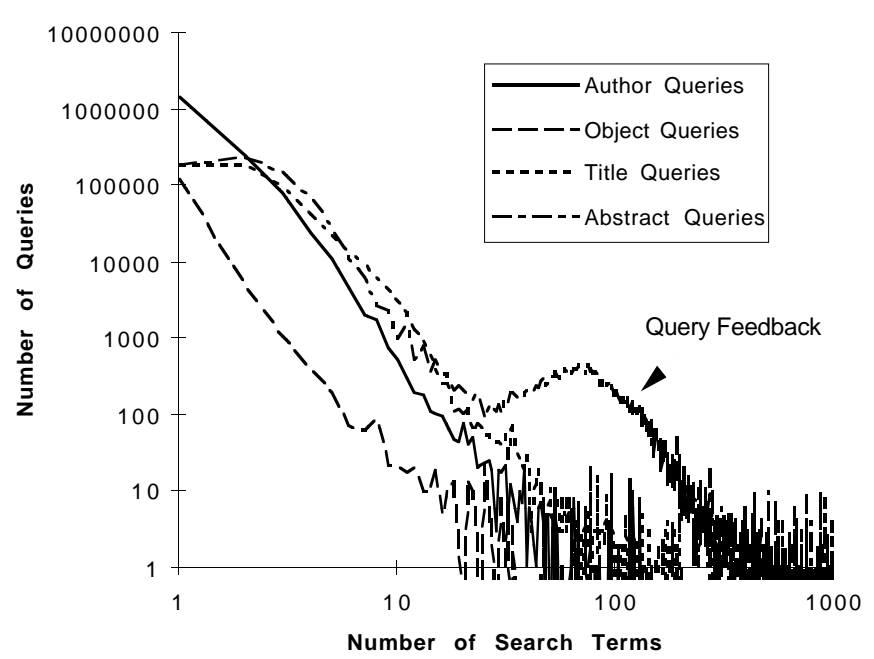

Fig. 14. The number of queries in the period of 1 April 1998 to 31 March 1999 as a function of the number of query items in each input field

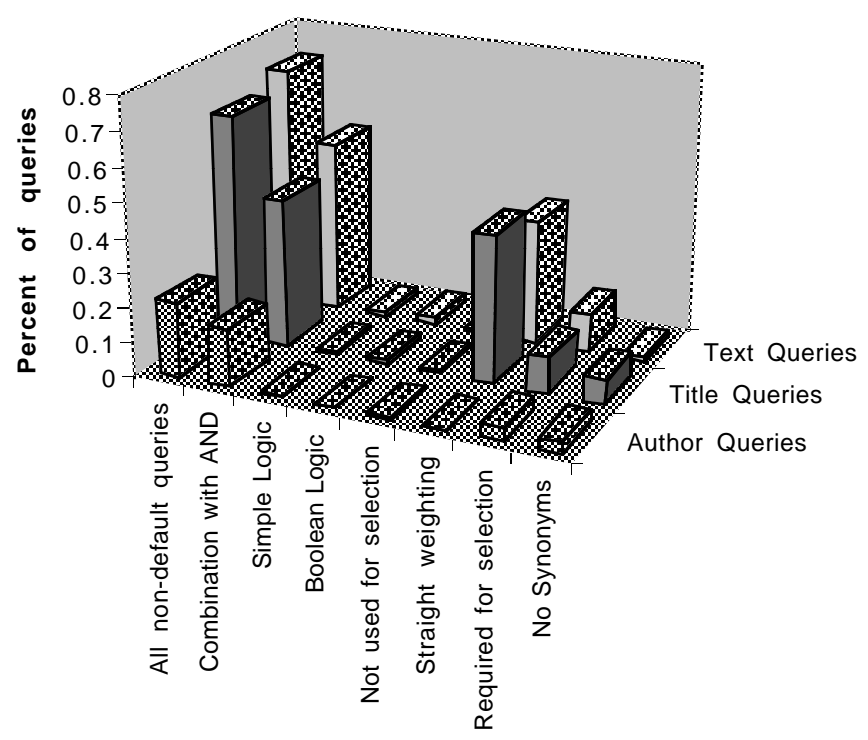

Fig. 15. Percentage of non-default settings for the different available settings and query fields

chosen to suffice for most queries. Figure 15 shows the percentage of non-default settings for the different settings and query fields available. It shows that $29 \%$ of author queries, $78 \%$ of title queries, and $85 \%$ of text queries use non-default settings. This was at first disappointing, because it suggested that the default settings might not be a reasonable selection of settings. The two main settings that were non-default were combining words with "AND" (see Sect. 2.1.1.b.ii), and disabled weighted scoring (see Sect. 2.1.1.c). On closer examination of the statistics it turns out that the straight weighting settings come from mainly two systems, the NASA Techreports and the International Society for Optical Engineering (SPIE).

Both of these systems use our Perl scripts (see Sect. 2.4) to access the ADS database. They do not

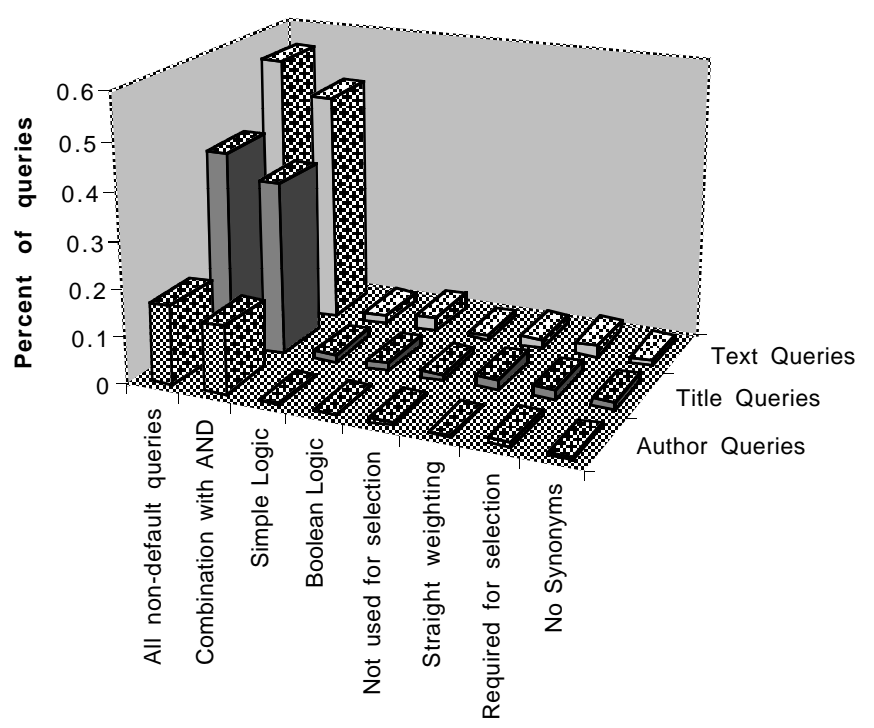

Fig. 16. Percentage of non-default settings for the different available settings and query fields. This plot excludes the queries from NASA Techreports and SPIE

set our normal default settings during these queries. Figure 16 shows the non-default settings for all queries that did not come from either of these two servers. There is still a small percentage of queries that use straight weighting, probably mostly due to other systems that use our Perl script interface routines.

The one remaining non-default setting that is used frequently is the combination of words with "AND". We believe that the "OR" combination as default is more useful since it returns more information. The beginning of the list of returned references is the same, regardless of whether "AND" or "OR" combination is selected, since references that match all words are sorted to the beginning of the list. When "OR" combination is selected, partial matches will be returned after the ones with perfect matches. This is desirable since there may be relevant references that for some reason do not match all query words.

The other selecting mechanism that is available is the filtering of references according to what other information is available for a reference. The usage of the filtering is shown in Table 8 . About $10 \%$ of the total queries use the filter option. Almost all of these filter by journal or select refereed journals only. The sum of the numbers for required data types adds up to more than the number for "Required data", since more than one data type can be selected.

Table 9 shows the number of links available and the usage pattern of the data links that the ADS provides. The highest usage is access to the abstracts, followed by the links to full text articles, links to citations, and links to on-line electronic articles. Reference links and links to SIMBAD objects are next. 
Table 8. Filter requests during the period of 1 April 1998 to 31 March 1999

\begin{tabular}{|c|c|c|}
\hline Filter Type & Required Data Type & Queries \\
\hline Total queries & & 2754405 \\
\hline Non-standard queries & & 286341 \\
\hline Selected journal & & 158581 \\
\hline Refereed journals & & 96270 \\
\hline Non-refereed journals & & 1616 \\
\hline Data available & & 6381 \\
\hline \multicolumn{3}{|l|}{ Required data } \\
\hline & Printable Articles & 2921 \\
\hline & Scanned Articles & 1951 \\
\hline & Electronic Articles & 1690 \\
\hline & Abstracts & 1382 \\
\hline & Planetary Data System & 834 \\
\hline & Planetary Nebulae & 667 \\
\hline & Citations & 615 \\
\hline & Table of Contents & 506 \\
\hline & References & 459 \\
\hline & Author Comments & 393 \\
\hline & On-line Data & 360 \\
\hline & SIMBAD Objects & 269 \\
\hline & NED Objects & 212 \\
\hline & Library Entries & 204 \\
\hline & Mail Order & 201 \\
\hline & Associated Articles & 83 \\
\hline
\end{tabular}

Table 9. Link types and their accesses during the period of 1 April 1998 to 31 March 1999. Numbers of links available are as of July 1999

\begin{tabular}{lrr}
\hline Links & Nr. Links & Nr. Accesses \\
\hline Abstracts & 941,293 & $1,608,726$ \\
Scanned Articles & 138,785 & 526,872 \\
Printable Articles & 40,928 & 254,881 \\
(Postscript and PDF) & & \\
Electronic Articles & 125,933 & 186,067 \\
Citations & 195,192 & 77,316 \\
References & 135,474 & 36,969 \\
SIMBAD Objects & 110,308 & 23,505 \\
On-line Data & 5,728 & 9,799 \\
NED Objects & 31,801 & 6,144 \\
Mail Order & 247,282 & 3,520 \\
Library Entries & 18,746 & 1,645 \\
Tables of Contents & 5,792 & 1,233 \\
Author Comments & 203 & 313 \\
Associated Articles & 2765 & 169 \\
Planetary Nebulae Data & 281 & 143 \\
\hline
\end{tabular}

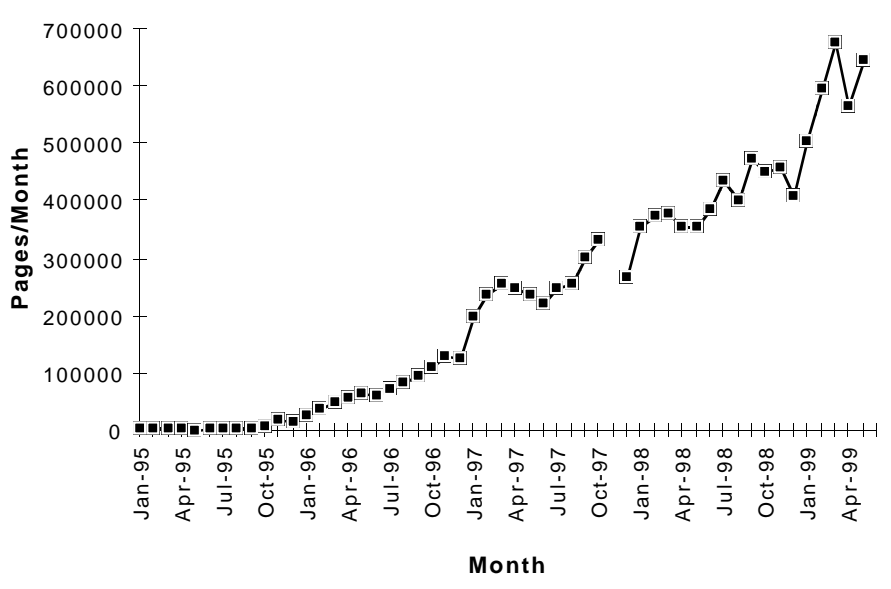

Fig. 17. Number of pages of scanned articles retrieved through the life of the ADS Article Service

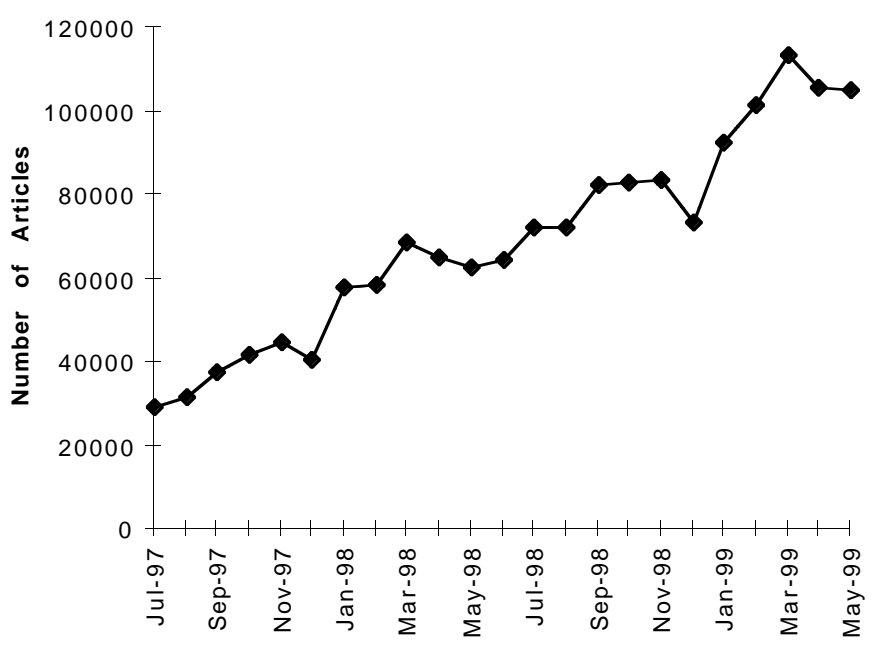

Fig. 18. Number of full text articles retrieved by ADS users. These numbers include the scanned articles at the ADS, as well as articles at the sites of the different journals that were requested through ADS links

\subsection{Article access statistics}

The ADS Article Service provides access to full journal articles. The usage statistics should show how astronomy researchers read and use journal articles. In this section we describe a few of the statistics of the article server. More statistics on the usage of the scanned articles are described in OVERVIEW.

Figure 17 shows the number of pages of scanned articles retrieved over the life of the ADS, Fig. 18 shows the number of articles retrieved. The number of articles represents the sum of the selected links to on-line electronic articles, PDF and Postscript articles at the journals, and scanned articles at the ADS.

Both the number of pages and the number of articles retrieved is steadily increasing. This is due to both the increased coverage in the ADS of scanned journals and the increase in the number of users that use the system. 
Table 10. Article retrieval by format type for March 1999, March 1998, and March 1997. PDF format and GIF Thumbnails were not yet available in March 1997

\begin{tabular}{lrrr}
\hline Article Type & \multicolumn{3}{c}{ Number of Retrievals } \\
& March 99 & March 98 & March 97 \\
\hline $\begin{array}{l}\text { Postscript Level 1 } \\
\text { (Low Resolution) }\end{array}$ & 476 & 557 & 644 \\
$\begin{array}{l}\text { Postscript Level 2 } \\
\text { (Low Resolution) }\end{array}$ & 25664 & 13031 & 11189 \\
$\begin{array}{l}\text { Postscript Level 2 } \\
\text { (High Resolution) }\end{array}$ & 10472 & 8291 & 6435 \\
$\begin{array}{l}\text { PDF } \\
\text { (Low Resolution) }\end{array}$ & 3266 & 620 & n/a \\
$\begin{array}{l}\text { PDF } \\
\text { (High Resolution) }\end{array}$ & 7049 & 1008 & n/a \\
$\begin{array}{l}\text { PCL } \\
\text { (Low Resolution) }\end{array}$ & 14 & 73 & 72 \\
$\begin{array}{l}\text { PCL } \\
\text { (High Resolution) }\end{array}$ & 53 & 111 & 132 \\
GIF Thumbnails & 13777 & 7378 & n/a \\
\hline
\end{tabular}

Table 10 shows the number of retrievals in the various formats. Postscript is a printer control language developed by Adobe (see Adobe Postscript 1990). Postscript Level 1 is the first version of the Postscript language. It generates much larger files than Level 2 Postscript. Some older printers can process only Level 1 Postscript files. PDF (Portable Document Format) is a newer page description format, also developed by Adobe. PCL (Printer Control Language) is a printer control language developed by Hewlett Packard. It is used in low end PC printers. Low resolution is 200 dpi for Postscript and PDF, and $150 \mathrm{dpi}$ for PCL. High resolution is $600 \mathrm{dpi}$ for Postscript and PDF, 300 for PCL.

The majority of retrievals are of medium resolution Postscript files. This is the default setting in the ADS Article Service. The number of Postscript Level 1 articles (compatible with older printers, but much larger file sizes) retrieved is low compared with Level 2 Postscript articles, and slowly declining. The number of PCL articles retrieved is even smaller and also declining. The number of PDF articles retrieved was slowly increasing throughout 1998. It has increased much more rapidly in 1999. In early 1998 less than $15 \%$ of the high resolution articles were retrieved as PDF files. This fraction increased to $40 \%$ by March, 1999.

\section{Future plans}

The ADS Abstract Service is only seven years old, but it is already an indispensable part of the astronomical research community. We get regular feedback from our users and we implement any reasonable suggestions. In this section we mention some of the plans for improvements that we are currently working on to provide even more functionality to our users.

\subsection{Historical literature}

One important part of the ADS Digital Library will be the historical literature from the 19th century and earlier. This part of the early literature is especially suited for being on-line in a central digital library. It is not available in many libraries, and if available is often in dangerously deteriorating condition. The access statistics of the ADS show that even old journal articles are accessed regularly (see OVERVIEW). The ADS is working on scanning this historical literature through two approaches: Scanning the journals, and scanning the observatory literature.

1. We are in the process of scanning the historical journal literature. We already have most of the larger journals scanned completely. Table 11 shows how much we have scanned of each of the journals and conference proceedings series for which we have permission. The oldest journal we have scanned completely is the Astronomical Journal (Vol. 1, 1849). We plan to have the Monthly Notices of the Royal Astronomical Society on-line completely by early in 2000. After that we plan to scan the oldest astronomical journal, Astronomische Nachrichten (Vol. 1, 1821), Icarus, Solar Physics, Zeitschrift für Astrophysik, Bulletin of the American Astronomical Society, and L'Observateur, and the conference series of IAU Symposia. Other journals that we plan to scan are the other precursor journals for Astronomy \& Astrophysics, if we can obtain permission to do so. We will also scan individual conference proceedings for which we can obtain permission.

2. One very important part of the astronomical literature in the 19th century and earlier were the observatory publications. Much of the astronomical research before the 20 th century was published in such reports. We are currently collaborating with the Harvard library in a project to make this part of the astronomical literature available through the ADS. The Harvard library has a grant from the National Endowment of the Humanities to make preservation microfilms of this (and other) literature. This project is generating an extra copy of each microfilm. We will scan these microfilms and produce electronic images of all the microfilmed volumes. The resolution of the microfilm and the scanning process is approximately equivalent to our 600 dpi scans. This project, once completed, will provide access to all astronomical observatory literature that is available at the Harvard library and the library of the Smithsonian Astrophysical Observatory from the 19th century back. For the more recent observatory literature we will have to resolve copyright issues before we can put it on-line. 
Table 11. Scanned journals in the ADS database

\begin{tabular}{lll}
\hline Journal & Scanned Volumes & Publication Dates \\
\hline Acta Astronomica & $42-48$ & $1992-1998$ \\
Annual Reviews of Astronomy and Astrophysics & $1-33$ & $1962-1995$ \\
Annual Reviews of Earth and Planetary Sciences & $1-23$ & $1973-1995$ \\
Astronomical Journal & $1-114$ & $1849-1997$ \\
Astronomical Society of the Pacific Conference Series & $1-5,7-22,24-63,65-69$ & $1988-1994$ \\
Astronomy and Astrophysics & $1-316$ & $1969-1996$ \\
Astronomy and Astrophysics Supplement Series & $1-120$ & $1969-1996$ \\
Astrophysical Journal & $1-473$ & $1895-1996$ \\
Astrophysical Journal Letters & $148-473$ & $1967-1996$ \\
Astrophysical Journal Supplement Series & $1-107$ & $1954-12 / 1996$ \\
Baltic Astronomy & $1-5$ & $1992-1996$ \\
Bulletin Astronomique de Belgrade & $153-155$ & $1996-1997$ \\
Bulletin of the Astronomical Institute of Czechoslovakia & $5-6,9-42$ & $1954-1955,1958-1991$ \\
Bulletin of the Astronomical Society of India & $8-23$ & $1980-1995$ \\
Journal of the Korean Astronomical Society & $1-29$ & $1968-1996$ \\
Journal of the British Astronomical Association & $92-107$ & $1981-1997$ \\
Meteoritics and Planetary Science & $1-33$ & $1953-1998$ \\
Monthly Notices of the Royal Astronomical Society & $1,100-301$ & $1827,1950-12 / 1998$ \\
AAS Photo Bulletin & $1-43$ & $1969-1986$ \\
Publications of the Astronomical Society of Australia & $1,3-13$ & $1967,1976-1996$ \\
Publications of the Astronomical Society of Japan & $1-50$ & $1949-1998$ \\
Publications of the Astronomical Society of the Pacific & $1-109$ & $1889-1997$ \\
Reviews in Modern Astronomy & $1-9$ & $1988-1996$ \\
Revista Mexicana de Astronomia y Astrofisica & $1-32$ & $1974-1996$ \\
Revista Mexicana de Astronomia y Astrofisica Ser. de Conf. & $1-6$ & $1995-1997$ \\
Skalnate Pleso, Contributions & $11-14,16-19,21-28$ & $1983-1986,1987-1990,1991-1998$ \\
\hline
\end{tabular}

In order to complete our data holdings, we still need issues of several journals for scanning. A list of journals and volumes that we need is on-line at:

http: //adsabs.harvard.edu/pubs/ missing_journals.html

In order to feed the pages through a sheet feeder, we need to cut the back of the volumes to be scanned. If they have not been bound before, they can be bound after the scanning. If they have been bound before, there is not enough margin left to bind them again. If you have any of the journals/volumes that we need, and you are willing to donate them to us, please contact the first author of this article. We would like to have even single volumes of any of the missing journals.

\subsection{Search capabilities}

The capabilities of the search system and user interface have been developed in close cooperation with our users. We always welcome suggestions for improvements and usually implement reasonable suggestions very quickly (within days or a few weeks). Because of this rapid implementation of new features we have hardly any backlog of improvements that we want to implement. There are currently two larger projects that we are investigating.
1. We plan to convert all our scanned articles into electronic text through Optical Character Recognition (OCR). In order to be able to search full text articles we will need to develop new search algorithms. Our current search system depends on the abstracts being of fairly uniform length. This caused some problems at one time when we included sets of data with abstracts that were $4-5$ times as long as our regular abstracts. These long abstracts would be found disproportionally often in searches with many query words (for instance in query feedback searches), since they generally matched more words. We had to reduce the sizes of these abstracts in order to make the searching work consistently with these data sets. OCR'd full text will require new search algorithms to make the search work at all. We currently estimate that the implementation of the full text search capability will take at least 2 years.

2. The scanned articles frequently contain plots of data. For most of these plots the numerical data are not available. We plan to develop an interface that will allow our users to select a plot, display it at high resolution, and digitize the points in the plot by clicking on them. This will allow our users to convert plots into data tables with as much precision as is available on the printed pages. This feature has been made available after this article was accepted for publication. 


\subsection{Article access}

We are currently investigating several new data compression schemes that would considerably reduce the size of our scanned articles. This could considerably improve the utility of the ADS Article Service, especially on slow links. We plan to be quite conservative in our approach to this, since we do not want to be locked into proprietary compression algorithms that could be expensive to utilize. At this point we do not have any time frame in which this might be accomplished.

\section{Summary}

The ADS Abstract Service has been instrumental in changing the way astronomers search and access the literature. One of the reasons for the immediate acceptance of the ADS by the astronomical community when the WWW based version became available was the ease of use of the interface. It provided access to many advanced features, while still making it extremely easy to execute simple queries. Most of the use is for simple queries, but a significant number of queries utilize the more sophisticated capabilities.

The search engine that provides this access was crucial to the success of the ADS as well. The most important aspects of a search engine are its speed and its flexibility to accommodate special features. The custom-designed software of the ADS search engine proved that at least in some instances a custom design has considerable advantage over a general purpose system. The search speed that we were able to achieve and the flexibility of the custom design that allows us to quickly adapt to our users' needs have justified the efforts of developing a system from scratch that is tailored to the specific data set.

The ADS had 31533 users issue 780711 queries and retrieve 15238646 references, 615181 abstracts, and 1119122 scanned pages in November, 1999. Since the start of the ADS we have served 311594261 references and 17146370 scanned article pages. These numbers speak for them self about the success of the ADS.

Acknowledgements. Funding for this project has been provided by NASA under NASA Grant NCC5-189.

\section{References}

Accomazzi A., Grant C.S., Eichhorn G., Kurtz M.J., Murray S.S., 1996, ASP Conf. Ser. 101: Astronomical Data Analysis Software and Systems V, 558
Accomazzi A., Eichhorn G., Grant C.S., Kurtz M.J., Murray S.S., 2000, A\&AS 143, 85

Adobe Acrobat Reader, http://www.adobe.com/prodindex/acrobat/alternate.html

Adobe Systems 1990, "Postscript Language Reference Manual, Second Edition". Addison-Wesley, Reading, MA

CIA World Factbook, 1999, US Government Publications, http://www.cia.gov/cia/publications/factbook/

Egret D., Wenger M., Dubois P., 1991, The SIMBAD Astronomical Database, "Databases \& On-line Data in Astronomy", Egret D. \& Albrecht M. (eds.). Kluwer Acad. Publ., p. 79

Eichhorn G., 1994, Exp. Astron. 5, 205

Eichhorn G., Accomazzi A., Grant C.S., Kurtz M.J., Murray S.S., 1995, Vistas Astron. 39, 217

Eichhorn G., Murray S.S., Kurtz M.J., Accomazzi A., Grant C.S., 1995, ASP Conf. Ser. 77: Astronomical Data Analysis Software and Systems IV, 28

Eichhorn G., 1996, Sky \& Telescope 92, 81

Eichhorn G., Accomazzi A., Grant C.S., Kurtz M.J., Murray S.S., 1996, ASP Conf. Ser. 101: Astronomical Data Analysis Software and Systems V, 569

Eichhorn G., 1997, Astrophys. Space Sci. 247, 189

Grant C.S., Eichhorn G., Accomazzi A., Kurtz M.J., Murray S.S., 2000, A\&AS 143, 111

Kristol D.M., Montulli L., "HTTP State Management Mechanism", RFC 2109, February, 1997

Kurtz M.J., Karakashian T., Grant C.S., Eichhorn G., Murray S.S., Watson J.M., Ossorio P.G., Stoner J.L., 1993, ASP Conf. Ser. 52: Astronomical Data Analysis Software and Systems II, 132

Kurtz M.J., Eichhorn G., Accomazzi A., Grant C.S., Murray S.S., 2000, A\&AS 143, 41

Lynch C.A., 1997, D-lib Magazine, Vol. 3, No. 4

Madore B.F., Helou G., Corwin H.G. Jr., Schmitz M., Wu X., Bennett J., 1992, ASP Conf. Ser. 25: Astronomical Data Analysis Software and Systems I, 47

Marsden B.G., 1980, Celest. Mech. 22, 63

Murray S.S., Brugel E.W., Eichhorn G., Farris A., Good J.C., Kurtz M.J., Nousek J.A., Stoner J.L., 1992, Astronomy from Large Databases II, 387

Salton G., McGill M.J., 1983, "Introduction to modern information retrieval". New York, McGraw-Hill

Schatz B.R., Hardin J.B., 1994, Sci 265, 895-901

Schmitz M., Helou G., Dubois P., Lague C., Madore B., Corwin H.G. Jr., Lesteven S., 1995, "Information \& On-line Data in Astronomy", Egret D. \& Albrecht M.A. (eds.). Kluwer Acad. Publ., p. 259

Vaudreuil G., 1992, "MIME: Multi-Media, Multi-Lingual Extensions for RFC 822 Based Electronic Mail" ConneXions, pp. 36-39

World Wide Web Consortium, 1999, http://www.w3.org 